\title{
How non-adiabatic passing electron layers of linear microinstabilities affect turbulent transport
}

\author{
J Dominski, ${ }^{1, \text { a) }}$ S Brunner, ${ }^{1}$ T Görler, ${ }^{2} \mathrm{~F}$ Jenko, ${ }^{2,3,4} \mathrm{D}$ Told, ${ }^{2,4}$ and L Villard ${ }^{1}$ \\ 1) École Polytechnique Fédérale de Lausanne (EPFL), Centre de Recherches en Physique des Plasmas (CRPP), \\ CH-1015 Lausanne, Switzerland. \\ ${ }^{2)}$ Max-Planck-Institut für Plasmaphysik, Boltzmannstraße 2, 85748 Garching, \\ Germany. \\ 3) Max-Planck/Princeton Center for Plasma Physics \\ ${ }^{4)}$ Department of Physics and Astronomy, University of California, Los Angeles, \\ California 90095.
}

(Dated: 6 March 2015)

\begin{abstract}
The response of passing electrons in ion temperature gradient (ITG) and trapped electron mode (TEM) microturbulence regimes is investigated in tokamak geometry making use of the flux-tube version of the gyrokinetic code GENE. Results are obtained using two different electron models, fully kinetic and hybrid in which passing particles are forced to respond adiabatically while trapped are handled kinetically. Comparing linear eigenmodes obtained with these two models enables to systematically isolate fine radial structures located at corresponding mode rational surfaces, clearly resulting from the non-adiabatic passing electron response. Non-linear simulations show that these fine structures on the non-axisymmetric modes survive in the turbulent phase. Furthermore, through non-linear coupling to axisymmetric modes, they induce radial modulations in the effective profiles of density, ion/electron temperature and $E \times B$ shearing rate. Finally, the passing electron channel is shown to significantly contribute to the transport levels, at least in our ITG case. Also shown is that the passing electrons significantly influence the $E \times B$ saturation mechanism of turbulence fluxes.
\end{abstract}

\section{INTRODUCTION}

In axisymmetric, magnetic fusion-type plasmas such as generated in tokamaks, the amplitude of the magnetic field roughly varies as the inverse of the distance $\mathrm{R}$ to the axis of symmetry, $B \propto 1 / R$, such that particles can be mirror-trapped in the low field side of the torus. Phase space of each plasma species (electrons and ions) can thus be separated into trapped and passing regions. When studying microinstabilities and associated turbulence at the ion time scale, as in the cases of ion temperature gradient (ITG) and trapped electron mode (TEM), it is often assumed that the real frequency $\omega_{r}$ of these modes is sufficiently low compared to the fast parallel dynamics of the passing electrons for these passing electrons to respond adiabatically. The reduced adiabatic (Boltzmann) response $^{1}$ for passing electrons has thus been extensively applied in gyrokinetic codes with the purpose of studying turbulent transport in the ITG and TEM regimes, for example in ORB5 $5^{2,3}, \mathrm{GT}_{5} \mathrm{D}^{4}$, and GYSELA ${ }^{5}$. This approximation is practical due to the large time scale separation between the ion and electron dynamics, especially that of passing electrons. Resolving the full kinetic evolution of all species corresponds to a multi-scale computation which therefore remains a significant numerical challenge.

The assumption of an adiabatic response for the passing electrons is based on the argument that these particles are sufficiently mobile along the magnetic field line

\footnotetext{
a) julien.dominski@epfl.ch
}

to remain in thermal equilibrium even in the presence of field fluctuations ${ }^{1}$. This adiabatic response is justified as long as $\left|\omega_{r} / k_{\|}\right| \ll v_{\text {the }}$, i.e. the parallel phase velocity $\omega_{r} / k_{\|}$of ITG/TEM microinstabilities is small compared to the electron thermal velocity $v_{t h e}=\sqrt{T_{e 0} / m_{e}}$, where $k_{\|}$is the component parallel to the magnetic field of a given mode wave vector $\mathbf{k}, T_{e 0}$ the equilibrium temperature of the electrons, and $m_{e}$ their mass. Near mode rational surfaces (MRSs) of low order, i.e. magnetic surfaces where the safety factor $q_{s}$ is a low order rational number, $q_{s}=-m / n$ with $m, n$ integer, the adiabatic assumption is in fact not justified. Indeed, near such a MRS, resonant Fourier modes with poloidal and toroidal mode numbers $(m, n)$ align with the magnetic field line, $k_{\|} \approx\left(n q_{s}+m\right) / R q_{s}=0$. It results that the associated parallel phase velocity $\left|\omega_{r} / k_{\|}\right|$becomes larger than the electron thermal velocity $v_{\text {the }}$ within a certain radial width $\delta x$ around this surface. The condition for adiabatic response is thus clearly violated within this interval.

When accounting for the fully kinetic response of the electrons, fine structures related to their non-adiabatic response appear on the eigenmode structures in the vicinity of $\mathrm{MRSs}^{6,7}$. Their presence in non-linear simulations has been observed as well ${ }^{8-10}$, potentially altering the electron particle flux ${ }^{11}$. Turbulence simulation including full passing electrons dynamics has also been discussed in 12 . We propose here to more systematically characterize the role of the non-adiabatic response of passing electrons near MRSs, its linear destabilization mechanism, as well as its effect on the non-linear turbulent saturated regimes. We thus present the results of a systematic study of the non-adiabatic response of passing 
electrons in the vicinity of MRSs in both an ITG and a TEM dominated regimes. This analysis is based on results obtained with the gyrokinetic code $\mathrm{GENE}^{13}$. Although GENE is a very comprehensive code, which in particular has been generalized more recently to enable global (finite $\rho^{\star}$ ) simulations ${ }^{13-15}$, this study has made use of its flux-tube (i.e. local) limit. In the considered flux-tube geometry, the density $N_{j 0}$ and temperature $T_{j 0}$ of a given species $j$, as well as corresponding inverse radial gradient lengths $1 / L_{N_{j}}=-d \log N_{j 0} / d r$ and $1 / L_{T_{j}}=-d \log T_{j 0} / d r$ respectively, $r$ being a radial coordinate with units of length labeling the magnetic surfaces, are evaluated at a given flux-surface of minor radius $r_{0}$ and are assumed constant. The only radial variation of an equilibrium profile which is accounted for is the safety factor $q_{s}(r)$, whose variation is assumed linear and thus characterized by $q_{0}=q_{s}\left(r_{0}\right)$ and magnetic shear $\hat{s}=\left(r_{0} / q_{0}\right) d q_{s} /\left.d r\right|_{r_{0}}$, thereby providing the appropriate magnetic topology required to study the particular particle dynamics that may develop in the vicinity of low order MRSs. For the magnetic equilibrium, a tokamak geometry with circular concentric magnetic surfaces is chosen, as described by the so-called analytic ad hoc model ${ }^{16}$. Note that in this study we consider non-collisional plasmas for simplicity.

The paper is organized as follows. In Sec. II, the general aspects of the GENE code as well as its three different electron models are described : 1) a fully kinetic, 2) a fully adiabatic, and 3) a hybrid electron model in which the trapped particles are described kinetically, while the passing ones are forced to respond adiabatically. Then one illustrates the difference between these electron responses by comparing the linear spectra obtained from GENE using these three models, in two chosen reference cases: an ITG and a TEM regimes respectively. In Sec. III, the impact of the non-adiabatic response of passing electrons on the linear mode destabilization is addressed. Systematically comparing eigenmodes obtained from GENE linear simulations where the electron response is fully kinetic, with eigenmodes obtained from simulations where the electron response is hybrid, one clearly identifies a fine structure near MRS resulting from the non-adiabatic response of passing electrons ${ }^{17}$. The underlying mechanisms of the destabilization near MRS due to the non-adiabatic response of passing electrons are revealed by the derivation of a local dispersion relation to which GENE results are confronted. A systematic comparison based on the estimate of the fine radial structure width, within which the electrons respond non-adiabatically, is carried out by scanning physical parameters: the magnetic shear $\hat{s}$, the safety factor $q_{0}$, the electron to ion temperature ratio $\tau=T_{e 0} / T_{i 0}$, the ion to electron mass ratio $\mu=m_{i} / m_{e}$ and the wave vector in the binormal direction $k_{y} \rho_{i}$ (normalized to the ion Larmor radius). The interplay between the non-adiabatic response of passing electrons and the non-linear turbulence is analyzed in Sec. IV. The persistence in the turbulent regime of these fine structures on the considered $k_{y} \neq 0$ modes is first addressed. The resulting radial modulation of the axisymmetric mode $\left(k_{y}=0\right)$ due to the non-linear coupling with the $k_{y} \neq 0$ modes is shown. In particular, the development of corrugations in the flux-surfaceand time-averaged profiles is pointed out as already observed $^{8-10}$. The importance of the passing electron contribution to the different turbulent fluxes is quantified as well as their influence on the $E \times B$ saturation mechanism. Conclusions are finally drawn in Sec. V

\section{ELECTRON MODELS IMPLEMENTED IN GENE}

\section{A. The GENE code}

The code GENE ${ }^{13}$ evolves the distributions of an arbitrary number of different particle species by solving the associated gyrokinetic equations ${ }^{18}$. GENE makes use of an Eulerian-based representation and enables to carry out linear and non-linear simulations of microinstabilities and related turbulence in magnetic confinement devices. The system of gyrokinetic equations is closed by reduced Maxwell's equations for electromagnetic potentials: the quasi-neutrality (or Poisson's) equation is sufficient if only electrostatic fluctuations are considered, while Ampère's law is invoked if electromagnetic fluctuations are accounted for. More details on the system of gyrokinetic equations implemented in GENE can be found in Ref. 19.

In GENE, the configuration space is described with field-aligned coordinates $(x, y, z)$, which is a natural choice for representing the fluctuating fields of microturbulence, as they are themselves aligned with the equilibrium magnetic field $\mathbf{B}_{0}\left(\mathbf{B}_{0} \| \nabla x \times \nabla y\right)$. For a tokamak configuration, in terms of magnetic coordinates $(\psi, \chi, \varphi)$ $=$ (poloidal magnetic flux, straight field line poloidal angle, toroidal angle), one defines the radial coordinate $x=r-r_{0}$, with $r$ a function of $\psi$ with units of length and $r_{0}$ the center of the simulation domain. The binormal coordinate, also called field-line coordinate, is defined by $y=C_{y}\left[q_{s}(\psi) \chi-\varphi\right]$ and the "parallel" coordinate by $z=\chi$. One chooses $C_{y}=r_{0} / q_{0}$ such that $y$ acquires units of length as well.

In GENE, the radial and binormal directions are represented in Fourier space with periodic boundary conditions, using $n_{k_{x}}=n_{x}$ and $n_{k_{y}}=n_{y} / 2$ complex Fourier modes (invoking reality condition). Periodic boundary conditions are actually imposed in the radial direction and natural in the binormal one. In GENE, the "parallel" direction is represented in direct space with $n_{z}$ grid points and the boundary conditions in this direction are pseudo-periodic ${ }^{19,20}$ :

$$
\mathcal{A}(x, y, z+2 \pi)=\mathcal{A}\left(x, y-C_{y} q_{s} 2 \pi, z\right),
$$

where the periodicity of the poloidal direction $\mathcal{A}(\psi, \chi, \varphi)=\mathcal{A}(\psi, \chi+2 \pi, \varphi)$ in $(\psi, \chi, \varphi)$ coordinates has been translated in $(x, y, z)$ coordinates. In Fourier representation, this results in a coupling of the modes through 
the relation $\widehat{\mathcal{A}}\left(k_{x}, k_{y}, z+2 \pi\right)=\widehat{\mathcal{A}}\left(k_{x}+2 \pi \hat{s} k_{y}, k_{y}, z\right)$. Consequently $2 \pi \hat{s} k_{y}$ must be a harmonic of the fundamental $k_{x, \text { min }}=2 \pi / L_{x}$ and the radial size of the flux-tube must be $L_{x}=M / \hat{s} k_{y, \min }$ an integer number $\mathrm{M}$ times the distance between lowest order MRSs: $L_{L M R S}=1 / \hat{s} k_{y, \min }$.

A tokamak magnetic surface can be represented in the plane $(\chi, \varphi)$ with $\chi$ and $\varphi$ varying betwen 0 and $2 \pi$. In these coordinates, the magnetic field lines are straight lines of constant safety factor $q_{s}=\mathbf{B} \cdot \nabla \varphi / \mathbf{B} \cdot \nabla \chi$ and the flux-tube simulation domain covers a fraction of the magnetic surface which is bounded between two straight lines distant of $\Delta \varphi=2 \pi / n_{0}$ in the toroidal direction (constant $\chi$ ) and $n_{0}$ an integer. By definition of the $y$ coordinate, the box size in the binormal direction is then $L_{y}=2 \pi C_{y} / n_{0}$. When $n_{0}>1$, only a fraction of the magnetic surface is accounted for and the box length in the $y$-direction must be much longer than the typical correlation length of the turbulence $\left(\approx 10 \rho_{i}\right)$ to avoid unphysical reconnection of turbulence. A similar constraint has to be respected in the radial direction, i.e. $L_{x}$ much longer than the correlation length of the turbulence, because periodic boundary conditions are not natural in this direction. Finally, having $L_{z}=2 \pi$ the dimensions $L_{x} \times L_{y} \times L_{z}$ of the flux-tube simulation box representing the configuration space have been determined by the choice of physical and numerical parameters.

The considered velocity space coordinates are the gyrocenter variables $\left(v_{\|}, \mu\right)$, with $\mu=m v_{\perp}^{2} / 2 B_{0}$ the magnetic moment and $v_{\|}$, resp. $v_{\perp}$, the parallel, resp. perpendicular, components of velocity to the magnetic field. The numerical representation of these directions originally infinite are restricted to $-v_{\max }<v_{\|}<+v_{\max }$ and $0<\mu<\mu_{\max }=m v_{\max }^{2} / 2 B_{0}$ where typically $v_{\max }=4-5 v_{\text {th }}$. This species dependent velocity phase space is discretized with $n_{v_{\|}}$equidistant mesh points in the $v_{\|}$direction and with $n_{\mu}$ points, either equidistant or given by Gauss-Legendre integration points, in the $\mu$ direction. In this paper we use the thermal velocity definition: $v_{t h}=\sqrt{T_{0} / m}$, while the GENE code uses a different thermal velocity definition: $v_{T}=\sqrt{2 T_{0} / m}$.

\section{B. Electron Models}

The implementation of the hybrid electron model, a new feature in GENE, is briefly described here. It is a reduced model for the electron dynamics in which the trapped particles are solved kinetically, while the passing particles are forced to respond adiabatically. This model is only to be considered for representing electrostatic fluctuations at the ion scale, such as typically the ITG and TEM. Indeed, by neglecting the non-adiabatic response of passing-electrons, one excludes the dynamics essential to electron temperature gradient (ETG) modes and furthermore is unable to represent fluctuations in the electron current essential for describing electromagnetic modes. In the frame of the hybrid model, the only field equation that needs to be accounted for is therefore the quasi-neutrality equation (QNE):

$$
\sum_{j=\text { species }} q_{j} \delta N_{j}=0,
$$

where $\delta N_{j}$ stands for the perturbed density of species $j$. Species-dependent quantities are labeled with the subscript $j$ : electric charge $q_{j}$, mass $m_{j}$, background density $N_{j 0}$, background temperature $T_{j 0}$, perturbed density $\delta N_{j}$, perturbed temperature $\delta T_{j}$, and thermal velocity $v_{t h j}=\sqrt{T_{j 0} / m_{j}}(j=i$ ions and $j=e$ electrons $)$.

When describing a certain species, or sub-group of species, kinetically, the corresponding density fluctuation $\delta N_{j}^{\mathrm{kin}}$ is given within the gyrokinetic formalism by

$$
\begin{aligned}
\delta N_{j}^{\mathrm{kin}}(\mathbf{x})= & 2 \pi \int_{V} d \mu d v_{\|} \frac{B_{0 \|}^{\star}\left(\mathbf{x}, v_{\|}\right)}{m_{j}}\left\{\overline{\delta f}_{j}\left(\mathbf{x}, v_{\|}, \mu\right)\right. \\
& \left.-\frac{q_{j} f_{j 0}\left(\mathbf{x}, v_{\|}, \mu\right)}{T_{j 0}}[\phi(\mathbf{x})-\overline{\bar{\phi}}(\mathbf{x}, \mu)]\right\},
\end{aligned}
$$

where $V$ represents the domain in velocity space of the considered sub-group of particles, $f_{j 0}$ stands for the corresponding unperturbed distribution and $\delta f_{j}$ for the fluctuating part provided by the gyrokinetic equation, $B_{0 \|}^{\star} / m_{j}$ is the Jacobian relative to the velocity variables $\left(v_{\|}, \mu\right), B_{0 \|}^{\star}=B_{0}+\left(m_{j} v_{\|} / q_{j}\right)\left(\nabla \times \mathbf{b}_{0}\right) \cdot \mathbf{b}_{0}, \mathbf{b}_{0}=\mathbf{B}_{0} / B_{0}$, and $\phi$ is the perturbed electric potential. In Eq. (3), the charge density of each species is decomposed into two contributions: the gyrodensity, explicitly expressed in terms of $\overline{\delta f}_{j}$, and the polarization drift contribution, expressed in terms of $\phi$. The notation $\overline{\mathcal{A}}$ used in (3) stands for the so-called gyroaverage of a given field $\mathcal{A}(\mathbf{x})$ seen by a particle over one cyclotron period:

$$
\overline{\mathcal{A}}(\mathbf{x}, \mu)=\frac{1}{2 \pi} \oint d \theta \mathcal{A}(\mathbf{x}+\boldsymbol{\rho}(\mathbf{x}, \mu, \theta)),
$$

with $\boldsymbol{\rho}(\mathbf{x}, \mu, \theta)$ the gyrovector of the particle and $\theta$ the gyroangle. Furthermore $\overline{\overline{\mathcal{A}}}$ stands for the double gyroaveraging:

$$
\overline{\overline{\mathcal{A}}}(\mathbf{x}, \mu)=\oint \frac{d \theta}{2 \pi} \oint \frac{d \theta^{\prime}}{2 \pi} \mathcal{A}\left(\mathbf{x}+\boldsymbol{\rho}(\mathbf{x}, \mu, \theta)-\boldsymbol{\rho}\left(\mathbf{x}, \mu, \theta^{\prime}\right)\right),
$$

where the variation of the Larmor radius between the particle position and the guiding-center position has been neglected: $\|\boldsymbol{\rho}\|(\mathbf{x}) \approx\|\boldsymbol{\rho}\|(\mathbf{x}-\boldsymbol{\rho})$. In Eq. (3), the background distribution $f_{j 0}$ has in fact been assumed Maxwellian:

$$
f_{j 0}=\frac{N_{j 0}}{\left(2 \pi T_{j 0} / m_{j}\right)^{3 / 2}} \exp \left(-\frac{m_{j} v_{\|}^{2} / 2+\mu B_{0}}{T_{j 0}}\right) .
$$

When describing a certain (sub-group) of species, adiabatically, the corresponding, linearized, density fluctuation response $\delta N_{j}^{\text {ad }}$ reads:

$$
\delta N_{j}^{\mathrm{ad}}(\mathbf{x})=\alpha \frac{q_{j} N_{j 0}}{T_{j 0}}\left(\phi-\langle\phi\rangle_{\mathrm{FS}}\right)
$$


where $\alpha$ is the fraction of the species population which compose the considered sub-group and $\langle\cdot\rangle_{\mathrm{FS}}$ stands for the flux-surface averaging operator

$$
\langle\cdot\rangle_{\mathrm{FS}}=\lim _{\Delta \psi \rightarrow 0} \frac{1}{\Delta V} \int_{\Delta V} d V
$$

with $\Delta V$ the space volume between two consecutive magnetic surfaces $\psi$ and $\psi+\Delta \psi$.

Let us now briefly detail the particular cases of the three electron models.

\section{Fully kinetic}

In the case of the fully kinetic model, the perturbed density of each species is computed kinetically by integrating Eq. (3) over the whole velocity phase-space, i.e. $\int_{V=\text { all }} d v_{\|} d \mu=\int_{0}^{+\infty} d \mu \int_{-\infty}^{+\infty} d v_{\|}$. In this case the QNE reads:

$$
\begin{aligned}
& \sum_{\mathrm{j}=\mathrm{i}, \mathrm{e}} q_{j}\left\{2 \pi \int_{0}^{+\infty} d \mu \int_{-\infty}^{+\infty} d v_{\|} \frac{B_{0 \|}^{\star}}{m_{j}} \overline{\delta f}_{j}\right. \\
& \left.-\frac{q_{j} N_{j 0}}{T_{j 0}}\left[\phi-\frac{B_{0}}{T_{j 0}} \int_{0}^{+\infty} d \mu \overline{\bar{\phi}} \exp \left(-\frac{\mu B_{0}}{T_{j 0}}\right)\right]\right\}=0,
\end{aligned}
$$

where the background Maxwellian distribution appearing in the polarization-drift term has been analytically integrated over the $v_{\|}$variable. Eq. (9) requires the evolution of both the ion and electron distributions.

\section{Adiabatic}

In the adiabatic model, all types of electrons are forced to respond adiabatically. Their perturbed density being thus determined with Eq. (7) where $\alpha$ is set to unity as the whole electron population is considered to respond adiabatically. In this case, the QNE reads:

$$
\begin{aligned}
& \sum_{i} q_{i}\left\{2 \pi \int_{0}^{+\infty} d \mu \int_{-\infty}^{+\infty} d v_{\|} \frac{B_{0 \|}^{\star}}{m_{i}} \overline{\delta f}_{i}\right. \\
- & \left.\frac{q_{i} N_{i 0}}{T_{i 0}}\left[\phi-\frac{B_{0}}{T_{i 0}} \int_{0}^{+\infty} d \mu \overline{\bar{\phi}} \exp \left(-\frac{\mu B_{0}}{T_{i 0}}\right)\right]\right\} \\
= & q_{e}^{2} \frac{N_{e 0}}{T_{e 0}}\left(\phi-\langle\phi\rangle_{\mathrm{FS}}\right),
\end{aligned}
$$

where the sum over species on the left hand side of Eq. (9) is now reduced to the sole (multiple) ion contribution(s), while the charge density contribution from the adiabatic electron response appears on the right hand side of Eq. (10). For this adiabatic model, there is clearly no need to evolve the gyrokinetic equation for the electron distribution.

\section{Hybrid}

Finally, in the case of the hybrid model, the QNE is written:

$$
\begin{aligned}
& \sum_{i} q_{i}\left\{2 \pi \int_{0}^{+\infty} d \mu \int_{-\infty}^{+\infty} d v_{\|} \frac{B_{0 \|}^{\star}}{m_{i}} \overline{\delta f_{i}}\right. \\
- & \left.\frac{q_{i} N_{i 0}}{T_{i 0}}\left[\phi-\frac{B_{0}}{T_{i 0}} \int_{0}^{+\infty} d \mu \overline{\bar{\phi}} \exp \left(-\frac{\mu B_{0}}{T_{i 0}}\right)\right]\right\} \\
= & \left(1-\alpha_{t}\right) q_{e}^{2} \frac{N_{e 0}}{T_{e 0}}\left(\phi-\langle\phi\rangle_{\mathrm{FS}}\right) \\
- & 2 \pi q_{e} \int_{0}^{+\infty} d \mu \int_{-v_{\| c}}^{+v_{\| c}} d v_{\|} \frac{B_{0 \|}^{\star}}{m_{e}} \overline{\delta f}+\frac{q_{e}^{2} N_{e 0}}{T_{e 0}} \alpha_{t} \phi \\
- & \frac{q_{e}^{2} N_{e 0} B_{0}}{T_{e 0}^{2}} \int_{0}^{+\infty} d \mu \overline{\bar{\phi}} \exp \left(-\frac{\mu B_{0}}{T_{e 0}}\right) \operatorname{erf}\left(\frac{v_{\| c}}{2 v_{t h e}}\right)
\end{aligned}
$$

where the fluctuating component of the electron charge density on the right hand side yields two contributions: the first term from the passing particles, handled adiabatically, and the other terms from the trapped particles, handled kinetically. The trapped electron contribution, at a position $\mathbf{x}$, is obtained by integrating the kinetic estimate as described in Eq. (3) over the velocity phase-space volume of trapped particles $\int_{V=\operatorname{trp}} d \mu d v_{\|}=$ $\int_{0}^{+\infty} d \mu \int_{-v_{\| c}}^{+v_{\| c}} d v_{\|}$which is characterized by the critical parallel velocity $v_{\| c}(\mathbf{x}, \mu)=\sqrt{2 \mu\left[B_{0, \max }(x)-B(\mathbf{x})\right] / m_{e}}$ where $B_{0, \max }(x)$ is the maximum of the magnetic field on the magnetic surface of radial position $x$ on which $\mathrm{x}$ lie. The passing electrons contribution, at the same position, is estimated by weighting Eq. (7) by the passing electron fraction $\alpha=\left(1-\alpha_{t}\right)$, itself defined by the trapped electron fraction $\alpha_{t}$ which can be estimated as

$$
\alpha_{t}=\int_{\mathrm{V}=\text { trap }} d^{3} v \frac{f_{e 0}}{N_{e 0}}=\sqrt{1-\frac{B_{0}}{B_{0, \max }(x)}} .
$$

In Eq. (11) the presence of the error function, $\operatorname{erf}(x)=$ $(2 / \sqrt{\pi}) \int_{0}^{x} d t e^{-t^{2}}$, results from the analytical integration of the background Maxwellian distribution appearing in the polarization drift term over the parallel velocity phase-space of trapped electrons. Note that in the limit of $v_{\| c} \rightarrow \infty\left(\alpha_{t} \rightarrow 1\right)$, one recovers the fully-kinetic model of Eq. (9) from Eq. (11). Respectively, in the limit of $v_{\| c} \rightarrow 0\left(\alpha_{t} \rightarrow 0\right)$, one recovers the adiabatic model of Eq. (10) from Eq. (11).

In the hybrid model, only the distribution of trapped electrons is required to evaluate the QNE. The distribution of passing electrons however does not explicitly appear in this equation. It may therefore appear that the passing electron distribution can be neglected. This is in fact not true, as the effective particle trajectories, perturbed by the fluctuating fields associated to the microturbulence, can lead to passing particles becoming trapped and vice versa. Consequently, the total electron distribution must nonetheless be evolved in the hybrid model, according to the same gyrokinetic equation 
(a) ITG, GENE

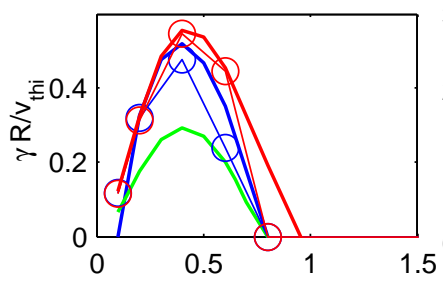

(c) ITG, GENE

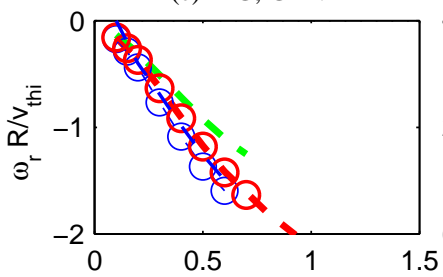

(e) ITG, local

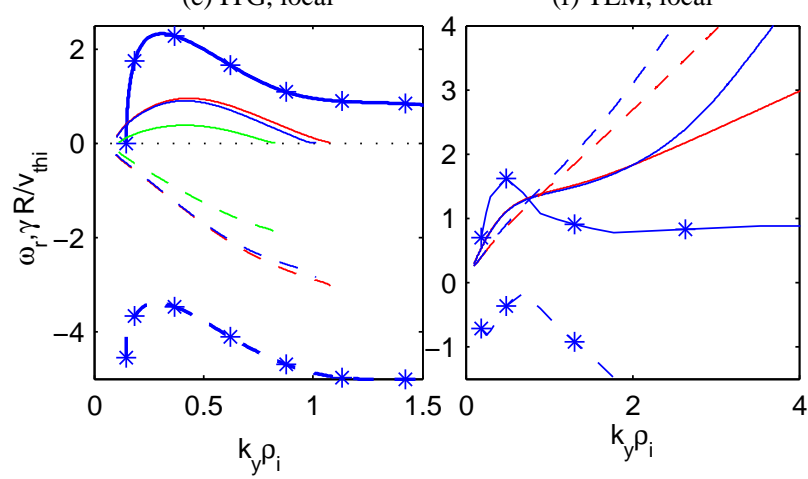

(b) TEM, GENE

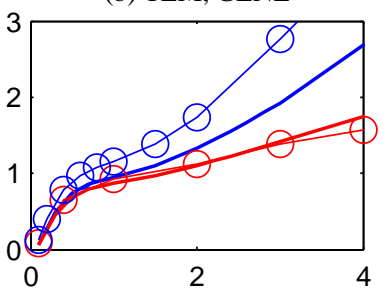

(d) TEM, GENE

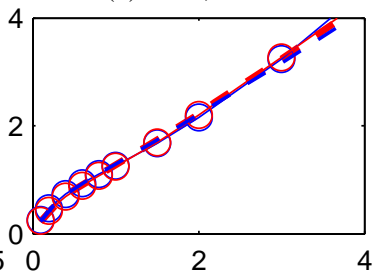

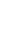

For all cases: concentric circular geometry with $q_{0}=1.4$, $\hat{s}=0.8, Z=1, \tau=T_{e 0} / T_{i 0}=1 . \beta=10^{-3}$ for fully-kinetic model.

\begin{tabular}{|l|c|c|c|c|c|c|}
\hline \hline Case & $R / L_{n}$ & $R / L_{T_{e 0}}$ & $R / L_{T_{i 0}}$ & $k_{y, \min } \rho_{i}$ & $\mu=m_{i} / m_{e}$ & $\epsilon=r_{0} / R$ \\
\hline ITG & 2.0 & 2.0 & 6.0 & $0.3(0.07)$ & $1836(400)$ & 0.18 \\
\hline TEM & 3.0 & 6.0 & 0.0 & $0.3(0.04)$ & $1836(400)$ & 0.16 \\
\hline
\end{tabular}

Grid resolution: $n_{x} \times n_{k_{y}} \times n_{z} \times n_{v_{\|}} \times n_{\mu}=64 \times 1 \times 32 \times 64 \times 32$ $(432 \times 64 \times 16 \times 64 \times 8$ in non-linear ITG and $256 \times 64 \times 16 \times 64 \times 16$ in non-linear TEM).

TABLE I. Physical parameters for the ITG and TEM reference cases, considered in GENE simulations and in the local dispersion relation. The parameters $\epsilon, q_{0}$, and $\hat{s}$ stand respectively for the inverse aspect ratio and the magnetic shear at the center of the flux-tube. Parameter values in parenthesis correspond to the ones considered in the non-linear simulations when different from the corresponding linear run values.

most unstable mode at each wave number $k_{y}(\sim$ average poloidal) are presented in Fig. 1 subplots (a,b,c,d). This figure shows the results obtained for two different instability regimes: an ITG and a TEM. These two cases are the reference cases considered in this entire work. The ITG is close to the cyclone base case (CBC), whose characteristic gradients lengths of density and ion/electron temperatures have been slightly modified to avoid the presence of secondary TEM or ETG modes. The TEM case is inspired by Ref. 21 but considering the temperature ratio $\tau=1$ instead of 3 : will be discussed in Sec. IV A, this change is made to obtain a TEM case whose turbulent fluxes are significantly saturated by the zonal flows (ZFs).

In the ITG case, Fig. 1 (a) and (c), for small wave numbers, $0<k_{y} \rho_{i} \lesssim 0.7$, the fully kinetic electron model (blue) provides an ITG instability $\left(\omega_{r}<0\right)$, well reproduced by the hybrid (red) but not so well reproduced by the adiabatic model (green): there is indeed a $\sim 50 \%$ lower $\gamma$ with this latter model, resulting from the trapped electrons being forced to respond adiabatically, when in fact they are essentially passive for this ITG. Accounting for the fully kinetic electron response provides essentially the same spectra as when accounting for the hybrid electron response, in this ITG case where no unstable TEM or ETG are present.

In the TEM case, Fig. 1 (b) and (d), the kinetic model (blue) provides a TEM instability $\left(\omega_{r}>0\right)$ well reproduced by the hybrid model (red) for small to intermediate wave numbers $0<k_{y} \rho_{i} \lesssim 1.5$. At high wave numbers, $k_{y} \rho_{i} \gtrsim 1.5$, the fully kinetic spectra shows a progressive transition of the TEM mode towards an ETG instability, at which point the hybrid representation starts to fail. The adiabatic electron model does not give any TEM instability because this response cannot be used to reproduce the TEM destabilization mechanism which is caused by a resonance between the perturbation and the toroidal precessional drift of the trapped electrons. 


\section{FINE STRUCTURES AT LOW ORDER MRS, IN LINEAR REGIMES}

\section{A. Analytical estimate of the radial width of fine structures}

An analytical estimate of the radial width $\delta x^{\text {th }}$ measuring the region centered around a MRS, within which the passing electron response is expected to be non-adiabatic, is derived. The considered MRS is assumed located at $x_{0}=0$ where $q\left(x_{0}\right)=q_{0}=-m / n$. The wave vector of the Fourier mode of poloidal-toroidal mode numbers $(m, n)$ is given by $\mathbf{k}=m \nabla \chi+n \nabla \varphi$ and its component parallel to $\mathbf{B}_{0}$ reads:

$k_{\|}=\mathbf{k} \cdot \frac{\mathbf{B}_{0}}{B_{0}}=\frac{\mathbf{B}_{0} \cdot \nabla \chi}{B_{0}}\left[m+n q_{s}(x)\right]=\frac{\mathbf{B}_{0} \cdot \nabla \varphi}{B_{0} q_{s}}\left[m+n q_{s}(x)\right]$,

having used $q_{s}=\mathbf{B}_{0} \cdot \nabla \varphi / \mathbf{B}_{0} \cdot \nabla \chi$, constant on a magnetic surface for the straight field line poloidal angle $\chi$. Near the MRS located at $x_{0}$, one clearly has $k_{\|} \rightarrow 0$ and the condition of validity for limiting the passing electron response to their adiabatic response, $\left|\omega_{r} / k_{\|}\right| \ll v_{\text {the }}$, breaks down. Note that the parallel component of the wave vector goes to zero as the mode aligns with the magnetic field at the position of the MRS.

To have an estimate of the parallel phase velocity $\omega_{r} / k_{\|}$with respect to $x$, one Taylor expands the safety factor and the parallel component of the wave vector around $x_{0}$ with respect to the small deviation $x=x-x_{0}$, leading to:

$$
q_{s}(x) \simeq q_{0}\left(1+\frac{\hat{s} x}{r_{0}}\right)
$$

and

$$
k_{\|}(x) \simeq \frac{\hat{s}}{q_{0}} \frac{x}{R} k_{y},
$$

with $k_{y}=-n q_{0} / r_{0}$ and $\mathbf{B}_{0} \cdot \nabla \varphi \approx B_{0} / R$. Then, assuming that the electrons respond non-adiabatically in the region where the phase velocity of the perturbation is larger than the electron thermal velocity, one estimates the boundaries of this region to be where $\left|\omega_{r} / k_{\|}\left( \pm \delta x^{\text {th }} / 2\right)\right|=v_{\text {the }}$. Finally, the distance between these boundaries located on both sides of the MRS gives the so-called "theoretical" estimate of the fine structure

$$
\frac{\delta x^{\text {th }}}{\rho_{i}}=2 \frac{\left|\omega_{r}\right|}{v_{t h i} / R} \frac{q_{0}}{\hat{s} \sqrt{\tau \mu} k_{y} \rho_{i}} .
$$

This theoretical estimate requires the knowledge of the real frequencies, $\omega_{r}$, which we take here from GENE linear simulations. For example, to estimate the theoretical width of the $k_{y} \rho_{i}=0.3$ mode of reference, the real frequencies of the ITG and TEM cases are taken from Fig. $1(\mathrm{c}, \mathrm{d}): \omega_{r} \approx-0.7 v_{t h i} / R$ and $0.6 v_{t h i} / R$ respectively. Then considering appropriate physical parameters from Tab. I, one roughly estimates the respective radial widths to be $\delta x^{\text {th }} \approx 0.21 \rho_{i}$ and $0.18 \rho_{i}$. This narrow region of non-adiabatic electron response motivates the use of high radial resolution in GENE simulations.

\section{B. Fine structures near MRS in linear GENE simulations}

Linear flux-tube simulations of ITG and TEM eigenmodes have been carried out by using the spectral approach provided in the GENE code, which enables one to compute dominant as well as sub-dominant unstable eigenmodes with complex frequency $\omega=\omega_{r}+\imath \gamma$, as detailed in Ref. 22-24. In the GENE representation, an eigenmode of a field $\mathcal{A}$ thus takes on the functional form:

$$
\mathcal{A}(x, y, z ; t)=\widehat{\mathcal{A}}(x, z) \exp \left[\imath\left(k_{y} y-\omega t\right)\right]
$$

The eigenmode is therefore essentially characterized by the complex amplitude $\widehat{\mathcal{A}}(x, z)$, which represents the slow spatial variation, the fast phase variation being contained in the factor $\exp \left(\imath k_{y} y\right)$. Figs. 2 and 3 show typical eigenmode envelopes of fluctuating fields of interest $(\phi, \delta N$, $\delta T_{e}$ and $\left.\delta T_{i}\right)$, for both the ITG and TEM test cases in respectively the left and right columns of these figures. In these figures the fields are weighted by the Jacobian $\mathcal{J}^{\text {xyz }}$.

In a linear flux-tube simulation, due to the imposed periodic boundary conditions in $x$, the system size $L_{x}$ along this direction must be taken as the distance between consecutive lowest order MRSs for the considered $k_{y}$ mode number: $1=-n\left(d q_{s} / d x\right) L_{x}=k_{y} \hat{s} L_{x}$, leading to $L_{x}=1 /\left(k_{y} \hat{s}\right)$. Furthermore the origin of the $x$ coordinates is always chosen to be located at MRS. For $k_{y} \rho_{i}=0.3$ and $\hat{s}=0.8$ considered here, one thus obtains $L_{x}=4.17 \rho_{i}$.

In Fig. 2, whose results are obtained with the fully kinetic electron model, the characteristic ballooned structures of ITG/TEM modes is recovered: the amplitude of the slow spatial envelope of the perturbation is systematically modulated in the $z \equiv \chi$ direction with a minimum located in the inner mid-plane at $z= \pm \pi$ and a maximum located in the outer mid-plane at $z=0$. Indeed the maximum of amplitude is positioned in both cases at the outer mid-plane of the tokamak where the interchange is unfavorable in the ITG case and where the trapped electrons are resonating with the perturbation in the TEM case. Remarkable on all fields, except $\delta T_{i}$, is the presence of a fine radial structure centered on the MRS located, as previously mentioned above, at $x=0$ where the non-adiabatic response of the passing electrons cannot be neglected.

To clearly identify the effect of the non-adiabatic response of passing electrons, the envelopes of the electric potential $|\phi|$ obtained with the hybrid model and with the fully kinetic model, respectively, are compared in Fig. 3. Subplots $(\mathrm{a}, \mathrm{b})$ present results carried out with the fully kinetic model, while subplots $(\mathrm{c}, \mathrm{d})$ show results obtained with the hybrid model. Note that the ballooned structure is recovered for the case of hybrid electron model. A notable difference, however, is the presence of the fine almost slab-like structure centered at the MRS $(x=0)$ in the eigenmodes from the fully kinetic model, which is absent in the hybrid model results, thus clearly identified 


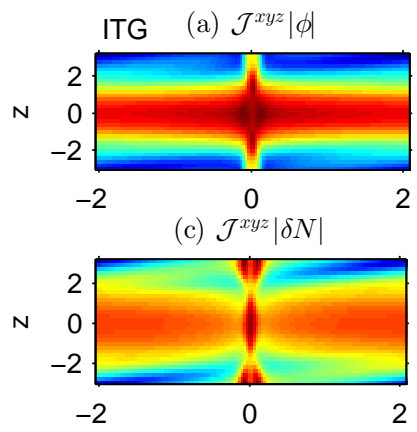

(e) $\mathcal{J}^{x y z}\left|\delta T_{e}\right|$

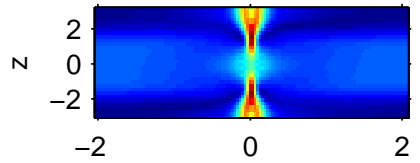

(g) $\mathcal{J}^{x y z}\left|\delta T_{i}\right|$
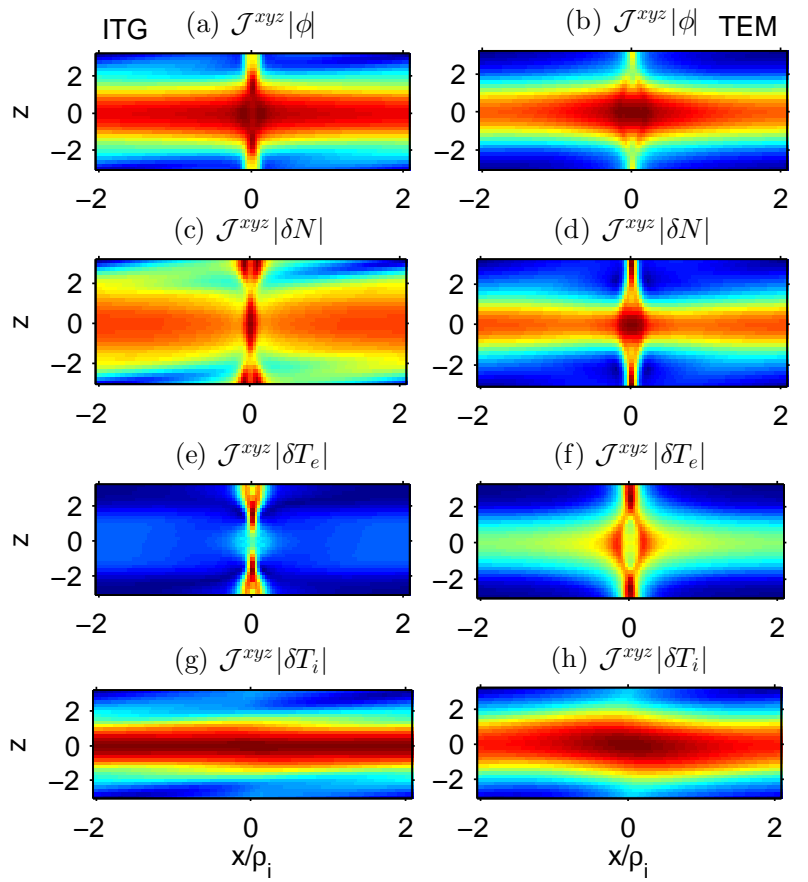

(f) $\mathcal{J}^{x y z}\left|\delta T_{e}\right|$

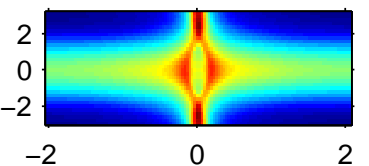

(h) $\mathcal{J}^{x y z}\left|\delta T_{i}\right|$

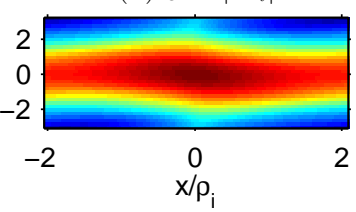

FIG. 2. $(x, z)$-envelopes of fluctuation fields $\phi, \delta N, \delta T_{e}, \delta T_{i}$ weighted by the Jacobian $\mathcal{J}^{\mathrm{xyz}}$ and corresponding to the ITG $(\mathrm{a}, \mathrm{c}, \mathrm{e}, \mathrm{g})$ and to the TEM (b,d,f,h) reference cases whose parameters are given in Tab. I. The fully kinetic model has been used. Color coding: dark blue $=$ zero, dark red = maximum value.

as a feature resulting from the non-adiabatic response of passing electrons in the vicinity of the lowest order MRS of the considered $k_{y}$ mode number. The slab-like feature along $z$ at $x=0$ ensures $\left|k_{\|}\right| \approx|\partial \log \widehat{\phi} / \partial z| \approx 0$, essential for a non-adiabatic response of passing electrons. $\partial \widehat{\phi} / \partial z=0$ is clearly only possible at MRS. By subtracting the mode envelopes obtained from the two different electron models, $\Delta|\widehat{\phi}|(x, z)=\left|\widehat{\phi}^{\text {kin }}\right|-\left|\widehat{\phi}^{\text {hyb }}\right|$ shown in subplots (e,f) of Fig. 3, we are able to cleanly isolate the radial structure. Note that prior to this subtraction, the field $\widehat{\phi}^{\text {hyb }}$ has been normalized such that $\left\langle\left|\widehat{\phi}^{\mathrm{hyb}}\right|\right\rangle_{z}=\left\langle\left|\widehat{\phi}^{\mathrm{kin}}\right|\right\rangle_{z}$ at $x= \pm L_{x} / 2$, i.e. at the most distant radial points from the MRSs where the fields provided by the fully kinetic and hybrid models are essentially identical. Furthermore $z$-averaging provides an average radial profile

$$
\langle\Delta|\widehat{\phi}|\rangle_{z}(x)=\frac{\int d z \mathcal{J}^{\mathrm{xyz}} \Delta|\widehat{\phi}|(x, z)}{\int d z \mathcal{J}^{\mathrm{xyz}}},
$$

shown in subplots $(\mathrm{g}, \mathrm{h})$ of Fig. 3, from which a numerical estimate $\delta x_{\text {lin }}^{\text {sim }}$ of the structure width is measured as the full width at half maximum (FWHM): $\delta x_{\operatorname{lin}}^{\sin } / \rho_{i} \approx$ 0.27 (ITG) and 0.48 (TEM). Numerical estimates are of the same order of magnitude than the theoretical ones, $\delta x^{\text {th }} / \rho_{i} \approx 0.21$ (ITG) and 0.18 (TEM), with good agreement for ITG and a factor 2.5 larger for TEM.

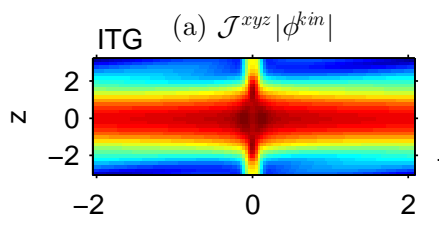

(c) $\mathcal{J}^{x y z}\left|\phi^{h y b}\right|$

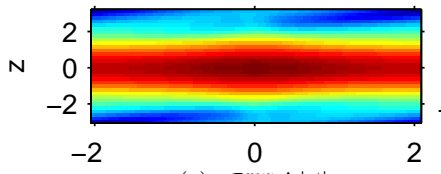

(e) $\mathcal{J}^{x y z} \Delta|\phi|$

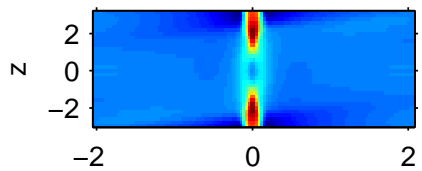

(g) $\langle\Delta|\phi|\rangle_{z}$
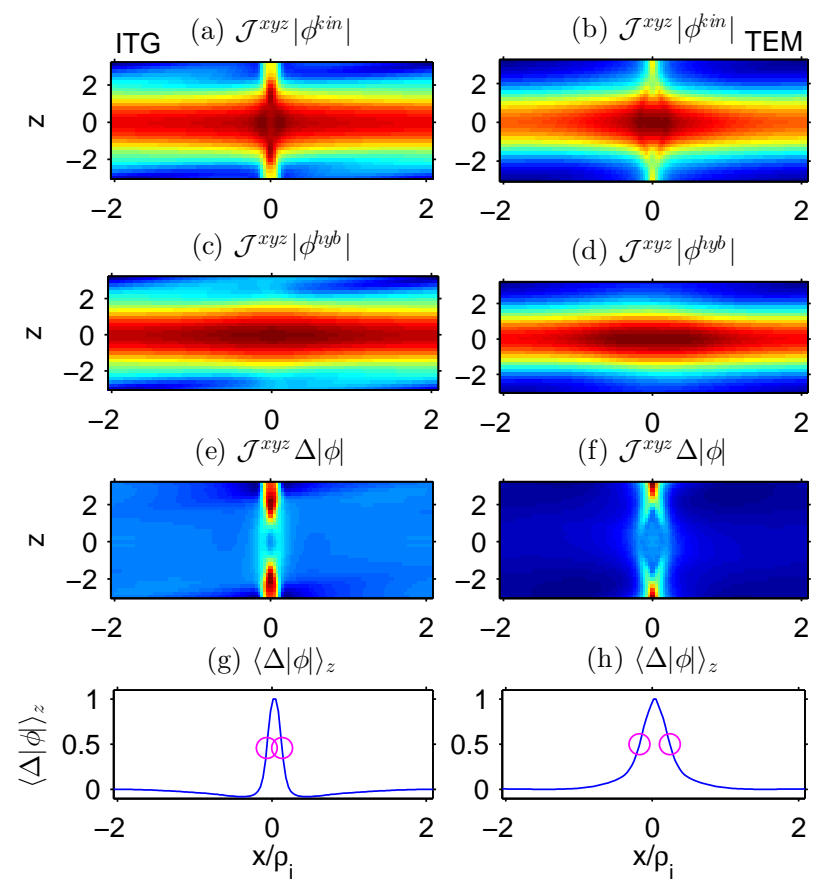

(d) $\mathcal{J}^{x y z}\left|\phi^{h y b}\right|$

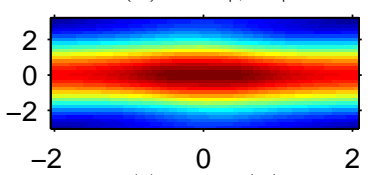

(f) $\mathcal{J}^{x y z} \Delta|\phi|$

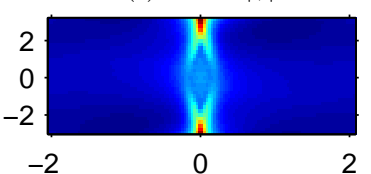

(h) $\langle\Delta|\phi|\rangle_{z}$

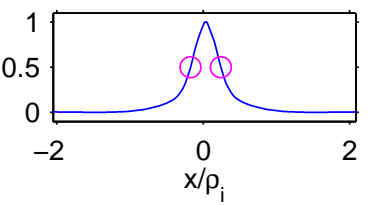

FIG. 3. Envelopes $\mathcal{J}^{\mathrm{xyz}}|\widehat{\phi}|(x, z)$ of linear eigenmodes for the same ITG and TEM cases as in Fig. 2. The first and second row of sub-plots respectively present the results from the fully kinetic and hybrid electron models. The difference $\Delta|\widehat{\phi}|$ is shown in the third row and its $z$-averaged profile in the fourth row, from which the numerical FWHM $\delta x_{\text {lin }}^{\text {sim }}$ (magenta circles) estimate for the fine structure width can be obtained. Color coding: dark blue $=$ zero, dark red $=$ maximum value

These fine structures can be indirectly observed through the presence of so-called "Giant tails" 11 in the ballooning representation of the electric potential. Indeed, giant tails in Fourier space correspond to a localized fine structure in direct space. Corresponding giant tails as illustrated in Fig. 4, for the ITG case, are present in the fully kinetic case, when no such big tails are present in the hybrid or adiabatic cases, thus confirming that the fine radial structures are due to the non-adiabatic response of the passing electrons. Nonetheless tails of lower amplitude (2 orders of magnitude smaller) are also present in the hybrid cases corresponding to weak radial modulation in direct space and visible in Fig 3(c-d). They are due to the trapped electrons response as there is no such tail of low amplitude in the adiabatic case. In the TEM case, not shown here, results are essentially the same.

\section{Local dispersion relation}

\section{Model}

In this section one introduces the local dispersion relation used to describe both ITG and TEM instabilities 


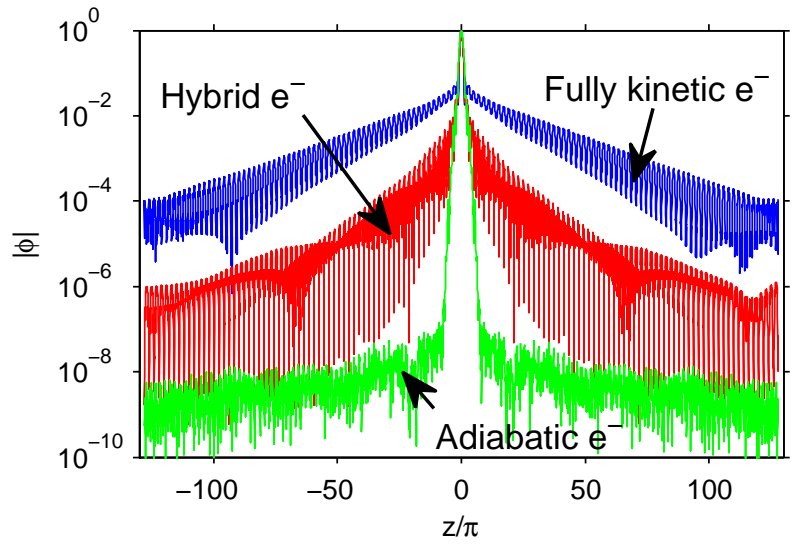

FIG. 4. Ballooning representation of the electric potential amplitude $|\phi|$ obtained in linear GENE simulations when using the different electron models. ITG test case with $n_{x}=128$.

at the most unfavorable position $(\chi=0)$. In this local approach the field aligned coordinate system is approximated by a local Cartesian system whose orthogonal directions are: the radial direction where the typical gradients are defined $\left(1 / L_{N, T_{i}, T_{e}}=d \ln \left(N, T_{i}, T_{e}\right) / d x\right)$, the binormal direction which is associated in this local approach to be the perpendicular direction $\left(k_{\perp} \equiv k_{y}\right)$, and the parallel direction to the magnetic field $\left(k_{\|} \equiv k_{z}\right)$. This dispersion relation is based on the gyrokinetic QNE. The perturbed density contribution of each species $j$ to the QNE is obtained from the gyrokinetic equation ${ }^{25}$ :

$$
\delta N_{j}=-\frac{q_{j} \phi}{T_{j 0}} \int_{V} d^{3} v\left[1-J_{0}^{2}\left(\vartheta_{j}\right) \frac{\omega-\omega_{j}^{\star}}{\omega-k_{\|} v_{\|}-\omega_{d j}}\right] f_{j 0},
$$

where the first term in the square bracket of the RHS gives the adiabatic response and the second term containing the Bessel function of the first kind $J_{0}$ gives the non-adiabatic response. Other parameters are: $\vartheta_{j}=$ $k_{\perp} v_{\perp} / \Omega_{j}, \Omega_{j}=q_{j} B_{0} / m_{j}$ the cyclotron frequency, $\omega_{d j}=$ $-\left(v_{\|}^{2}+v_{\perp}^{2} / 2\right) k_{\perp} / R \Omega_{j}$ the drift frequency related to curvature and gradient of $\mathbf{B}_{0}, \omega_{j}^{\star}=\omega_{N j}\left(1+\eta_{j} T_{j 0} \partial / \partial T_{j}\right)$, $\omega_{N j}=-T_{j 0} k_{\perp} / q_{j} B_{0} L_{N}$, and $\eta_{j}=L_{N_{j}} / L_{T_{j}}$. The kinetic estimate of the ion perturbed density with charge $q_{i}=Z e$ is obtained from Eq. (16) where the domain of integration is the whole velocity space, giving:

$\frac{\delta N_{i}}{N_{i 0}}=-\frac{Z e \phi}{T_{i 0}}\left[1-\int_{-\infty}^{+\infty} d^{3} v J_{0}^{2}\left(\vartheta_{i}\right) \frac{\omega-\omega_{i}^{\star}}{\omega-k_{\|} v_{\|}-\omega_{d i}} \frac{f_{i 0}}{N_{i 0}}\right]$.

In the frame of the hybrid electron model, the electron density is composed of two contributions: the adiabatic response of the passing electrons $\delta N_{e, p a s} / N_{e 0}=$ $\left(1-\alpha_{t}\right) e \phi / T_{e 0}$ and the bounce-averaged kinetic response of the trapped electrons

$$
\frac{\delta N_{e, t r p}}{N_{e 0}}=\alpha_{t} \frac{e \phi}{T_{e 0}}\left(1-4 \pi \int_{0}^{+\infty} d \varepsilon \sqrt{2 \varepsilon} \frac{\omega-\omega_{e}^{\star}}{\omega-n\langle\dot{\varphi}\rangle} \frac{f_{e 0}}{N_{e 0}}\right),
$$

where $\varepsilon=\left(v_{\perp}^{2}+v_{\|}^{2}\right) / 2$ is the kinetic energy divided by the mass and $n\langle\dot{\varphi}\rangle$ is the toroidal precessional drift frequency of the trapped electrons. Introducing the dispersion function $W(z)=(2 \pi)^{-1 / 2} \int_{\Upsilon} d x e^{-x^{2} / 2} x /(x-z)$, this last equation reduces ${ }^{26}$ to

$$
\frac{\delta N_{e, \operatorname{trp}}}{N_{e 0}}=\alpha_{t} \frac{e \phi}{T_{e 0}}\left\{1+\left(1-\frac{\omega_{e}^{\star}}{\omega}\right)\left[z_{b e}^{2} W\left(z_{b e}\right)\right]\right\},
$$

where $\omega_{\varphi e}=n\langle\dot{\varphi}\rangle v_{t h e}^{2} / \varepsilon=\omega_{N e} G L_{N} / R, G \approx 1$ and $z_{b e}=\operatorname{sgn}\left(\omega_{\varphi e}\right) \sqrt{2 \omega / \omega_{\varphi e}}$ has been chosen in order to respect causality over the Landau contour $\Upsilon$ of the integral defining $W(z)$.

In order to study the fully kinetic response of the plasma in this local approach, the previously ignored non-adiabatic response of the passing electrons is introduced. The passing electron drift frequency is averaged to zero $\omega_{d e}=0$ because these passing electrons circulate successively over the favorable and unfavorable sides of the poloidal plane with a much higher transit frequency $\omega_{t}$ than the typical ITG/TEM frequencies. The Larmor radius of electrons being small enough, $k_{\perp} \rho_{e} \ll 1$, the electron finite Larmor radius effects are neglected: $J_{0}\left(\vartheta_{e}\right) \approx 1$. The non-adiabatic response of passing electrons is then obtained by integrating Eq. (16) with $\omega_{d e}=0$ and $J_{0}=1$ over the passing velocity phase space $\int_{\text {pas }} d^{3} v=2 \pi \int_{-\infty}^{+\infty} d v_{\|} \int_{0}^{v_{\perp c}} d v_{\perp}$, where $v_{\perp c}=v_{\|} \tan \theta_{c}$ is the critical perpendicular velocity above which the electrons are trapped. Finally, the fully kinetic response of the passing electrons reads:

$$
\begin{aligned}
& \frac{\delta N_{e, \mathrm{pas}}}{N_{e 0}}=\left(1-\alpha_{t}\right) \frac{e \phi}{T_{e 0}} \\
& \quad \times\left\{1-\left(1-\frac{\omega_{e}^{\star}}{\omega}\right)\left[1-\frac{W\left(z_{e}\right)-\alpha_{t} W\left(z_{e} / \alpha_{t}\right)}{1-\alpha_{t}}\right]\right\},
\end{aligned}
$$

where $W(z)$ is the dispersion function and $z_{e}=\omega / k_{\|} v_{t h e}$. Finally, having a quasi-neutral background $\left(Z N_{i 0}=\right.$ $N_{e 0}$ ), the dispersion relation of the fully kinetic model is:

$$
\begin{aligned}
0 & =Z_{\tau}+1-Z_{\tau} \int d^{3} v J_{0}^{2}\left(\vartheta_{i}\right) \frac{\omega-\omega_{i}^{\star}}{\omega-k_{\|} v_{\|}-\omega_{d i}} \frac{f_{i 0}}{N_{i 0}} \\
& +\left(1-\frac{\omega_{e}^{\star}}{\omega}\right)\left[\alpha_{t} z_{b e}^{2} W\left(z_{b e}\right)\right] \\
& +\left(1-\frac{\omega_{e}^{\star}}{\omega}\right)\left[-1+\alpha_{t}+W\left(z_{e}\right)-\alpha_{t} W\left(\frac{z_{e}}{\alpha_{t}}\right)\right]
\end{aligned}
$$

Removing both terms proportional to $\left(1-\omega_{e}^{\star} / \omega\right)$ which contain the non-adiabatic response of electrons gives the dispersion relation of the adiabatic model. Removing only the second term proportional to $\left(1-\omega_{e}^{\star} / \omega\right)$ gives the dispersion relation of the hybrid model. 


\section{Analytical solution at MRS}

We are now interested in deriving an analytical solution at MRS, i.e. $k_{\|}=0$, by keeping the essential terms of this local dispersion relation. Eq. (21) is simplified by noting that $k_{\|} v_{\|} / \omega=0$. For all cases, ion finite Larmor radius effects turn out to be essential in the destabilization mechanism at MRS, and they are kept at second order by employing the approximation $J_{0}^{2}\left(\vartheta_{i}\right) \approx 1-\vartheta_{i}^{2} / 2$. This seems to be a reasonable approximation for our reference cases as $k_{\perp} \rho_{i}=0.3$. Also assuming $\left|\omega / \omega_{d i}\right| \gg 1$, the ion density response at MRS reads

$$
\frac{\delta N_{i}}{N_{i 0}} \approx-\frac{Z e \phi}{T_{i 0}}\left\{1-\frac{\omega-\omega_{i}^{\star}}{\omega}\left[1-\xi_{i}+\left(2-3 \xi_{i}\right) \frac{\left\langle\omega_{d i}\right\rangle}{2 \omega}\right]\right\},
$$

where $\xi_{i}=\left(k_{\perp} \rho_{i}\right)^{2}$ and $\left\langle\omega_{d i}\right\rangle=\int d v \omega_{d i} f_{i 0} / N_{i 0}=$ $-2 T_{i 0} k_{\perp} / q_{i} B_{0} R$ is the drift frequency moment of the Maxwellian distribution function. The kinetic response of the trapped electrons is assumed to be passive $(=0)$ in the ITG case and to be the bounce averaged one in the TEM case. The latter response is obtained by taking the asymptotic expansion $W\left(z_{b e}\right) \simeq-z_{b e}^{-2}-z_{b e}^{-4}$ when assuming $\left|z_{b e}\right| \gg 1$, thus giving ${ }^{26}$

$$
\frac{\delta N_{e, t r p}}{N_{e 0}} \simeq \alpha_{t} \frac{e \phi}{T_{e 0}}\left[\frac{\omega_{N e}-3 \omega_{\varphi e} / 2}{\omega}+\frac{3}{2}\left(1+\eta_{e}\right) \frac{\omega_{N e} \omega_{\varphi e}}{\omega^{2}}\right] .
$$

The kinetic response of passing electrons is obtained by making use of the asymptotic expansion $W\left(z_{e}\right) \simeq-z_{e}^{-2}$ for $\left|z_{e}\right| \gg 1$ which in the limit $k_{\|} \rightarrow 0$ leads to

$$
\frac{\delta N_{e, p a s}}{N_{e 0}} \underset{k_{\|} \rightarrow 0}{=}\left(1-\alpha_{t}\right) \frac{e \phi}{T_{e 0}} \frac{\omega_{N e}}{\omega} .
$$

These approximations finally lead to the so-called asymptotic dispersion relation

$$
\begin{aligned}
& 0=\omega^{2}\left(\xi_{i}+\frac{c_{1}}{Z \tau}\right) \\
& +\omega \quad\left\{\omega_{N i}\left[1-c_{2}-\left(1+\eta_{i}\right) \xi_{i}\right]-\left\langle\omega_{d i}\right\rangle\left(1-\frac{3}{2} \xi_{i}\right)\right\} \\
& \quad+\omega_{N i}\left\langle\omega_{d i}\right\rangle\left[\left(1+\eta_{i}\right)\left(1-\frac{3}{2} \xi_{i}\right)-\frac{3}{2} \eta_{i} \xi_{i}\right] \\
& \quad-c_{3} \omega_{N i}\left\{\omega-\frac{3 L_{N} G}{2 R}\left[\omega+Z \tau \omega_{N i}\left(1+\eta_{e}\right)\right]\right\},
\end{aligned}
$$

where $c_{1}$ is the proportion of adiabatic electrons, $c_{2}$ is the proportion of kinetic passing electrons, and $c_{3}$ is the proportion of kinetic trapped electrons. In the ITG case for adiabatic electrons $\left(c_{1}=1, c_{2}=0, c_{3}=0\right)$, for hybrid electrons $\left(c_{1}=1-\alpha_{t}, c_{2}=0, c_{3}=0\right)$, and for fully kinetic electrons $\left(c_{1}=0, c_{2}=1-\alpha_{t}, c_{3}=0\right)$. In the TEM case for the hybrid electron model $\left(c_{1}=1-\alpha_{t}, c_{2}=\right.$ $\left.0, c_{3}=\alpha_{t}\right)$ and for the fully kinetic model $\left(c_{1}=0, c_{2}=\right.$ $\left.1-\alpha_{t}, c_{3}=\alpha_{t}\right)$. Note that in all cases $c_{1}+c_{2}+c_{3}=1$ except in the ITG case for hybrid and fully kinetic models where $c_{1}+c_{2}+c_{3}=1-\alpha_{t}$ as trapped electrons are assumed to be passive. (a) ITG

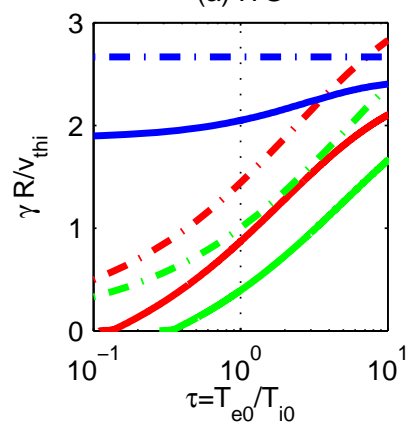

(b) TEM

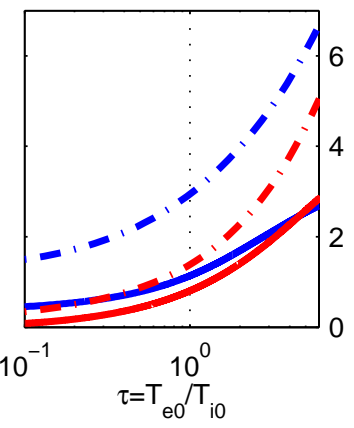

FIG. 5. Growth rate $\gamma$ at the MRS $\left(k_{\|}=0\right)$ obtained with the local dispersion relation Eq. (21) (full line), and with the asymptotic dispersion relation Eq. (25) (dash-dotted line). ITG case (a) and TEM case (b). Electron models: kinetic (blue), hybrid (red) and adiabatic (green). The vertical dotted line indicates the reference temperature ratio $\tau=1$.

The second order polynomial equation (25) of the form $A \omega^{2}+B \omega+C=0$ accounts for an instability if and only if the discriminant $\Delta=B^{2}-4 A C$ is negative. Noting that $A>0$, in all cases, a necessary condition for instability is thus $C>0$ which in turns requires $\omega_{N i}\left(1+\eta_{i}\right)\left\langle\omega_{d i}\right\rangle>0$ reflecting the interchange nature of the instability.

In order to appreciate the agreement between the local dispersion relation (21) and the asymptotic one (25), a scan in $\tau$ is carried out in Fig. 5. The destabilizing influence of the non-adiabatic electron response, near MRSs, is recovered with both dispersion relations: $\gamma^{\text {kin }}>\gamma^{\text {hyb }}>\gamma^{\text {ad }}$. The growth rates obtained from Eq. (25) are in a quite good agreement with those obtained from Eq. (21) in the ITG case but appear to be overestimated in the TEM case by a factor $\approx 2-3$ : this may be due to the approximation $\left|z_{b e}\right| \gg 1$ when in fact $\left|z_{b e}\right| \gtrsim 1$ in this TEM case when $k_{\|}=0$. Also for both local and asymptotic dispersion relations, increasing $\tau$, which corresponds to decreasing the adiabatic response, has the effect of reducing the difference between the growth rates obtained with the different electron models.

We now discuss results obtained with the local dispersion relation Eq. (21).

\section{Local destabilization near MRS, results}

The real frequencies and growth rates obtained with the local dispersion relation (21) are plotted in Fig. 1 subplots (e) and (f), in the ITG and TEM cases, respectively. To obtain an unstable branch from the local dispersion relation (21), one has to choose a value of the $k_{\|}$parameter. Results obtained with the adiabatic and hybrid models are not significantly affected by the value of $k_{\|}$and have then been plotted only for $k_{\|} R q_{0}=0.2$. On the contrary, results obtained with the fully kinetic 
model can be significantly affected by the value of $k_{\|}$especially if one compares cases for which $\omega_{r} / k_{\|} \ll 1$ or not -, results have thus been plotted for two values of $k_{\|}$. The first value of the parallel wave vector is the same as the one taken to obtain results with the hybrid and adiabatic models: $k_{\|} R q_{0}=0.2$; it corresponds to a value of the wave vector located far from a MRS where the adiabatic electron hypothesis is valid $\left(\left|\omega_{r} / k_{\|} v_{t h e}\right| \ll 1\right)$. The second value of the parallel wave vector is taken at MRS: $k_{\|} R q_{0}=0$, where the adiabatic hypothesis is invalid $\left(\left|\omega_{r} / k_{\|} v_{t h e}\right| \gg 1\right)$. Far from the MRS (full lines), the complex frequencies obtained with these local models are in a qualitative agreement with results obtained from GENE and plotted in subplots (a-d) but the local dispersion relation overestimates the real frequencies and growth rates up to a factor two. This difference can be explained by the fact that the local dispersion is located at the most unstable position, but the flux-tube accounts for both unstable and stable sides of the magnetic surface. At MRS (blue lines with asterisk), in comparison with kinetic results obtained far from MRS (blue lines), the ITG growth rates are larger for almost all wavelengths $\left(k_{y} \rho_{i} \gtrsim 0.1\right)$ and the TEM growth rates are larger for the long wavelengths $\left(k_{y} \rho_{i} \lesssim 0.8\right)$ and smaller elsewhere. This sensibility to the value of $k_{\|}$of the local dispersion relation with fully kinetic electrons underlines that the non-adiabatic response of passing electrons plays a role near MRS.

In Fig. 6 subplots (a,b) the real frequencies $\omega_{r}$ and growth rates $\gamma$ solutions of Eq. (21) are plotted with respect to $k_{\|}$, and in subplots $(\mathrm{c}, \mathrm{d})$ the phase to thermal velocities ratios $\left|\omega_{r} / k_{\|} v_{\text {the }}\right|$ are plotted with respect to $x$. The value of $x$ is computed using the mapping $k_{\|} \mapsto x$ described by Eq. (13). It clearly appears that when getting closer to the MRS, i.e. $k_{\|}$going to zero, the kinetic model provides larger growth rates than the hybrid model. It thus reveals the destabilizing role of the non-adiabatic response of passing electrons near MRS. Moreover, this divergence between kinetic and hybrid results starts from a value of $k_{\|}$where the parallel phase velocity of the wave is of the order of the electron thermal velocity. It validates the choice of the criteria $\left|\omega / k_{\|}\right|=v_{\text {the }}$ used to derive the theoretical width estimate of the fine structures Eq. (14).

In Fig. 7 the relative difference between kinetic and hybrid growth rates, $\Delta \gamma=\left(\gamma^{\text {kin }}-\gamma^{\text {hyb }}\right)$, obtained with the local dispersion relation, as well as the difference between kinetic and hybrid envelopes of the electric potential, $\Delta|\phi|=\left|\phi^{\text {kin }}\right|-\left|\phi^{\text {hyb }}\right|$, obtained with GENE are plotted with respect to the radial distance to the MRS. It is remarkable that the abrupt increase of the growth rate due to the non-adiabatic response of passing electrons near MRS occurs within a radial region where the radial fine structure is present over the perturbation fields of GENE results. Defining the local estimate of the fine structure width $\delta x^{\text {loc }}$ to be the FWHM of $\Delta \gamma(x)$, the structure boundary is then localized in Fig. 6 to be at $x / \rho_{i} \approx 0.1$ and 0.2 , in the ITG and TEM cases respectively. At these (a) ITG

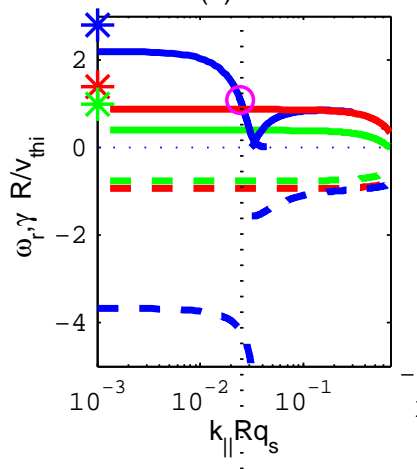

(c)

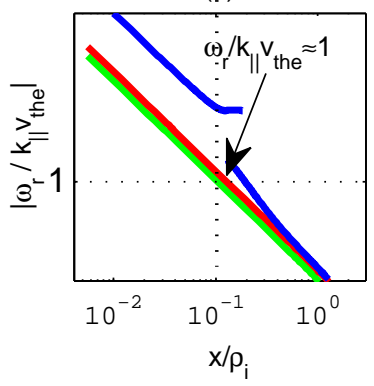

(b) TEM

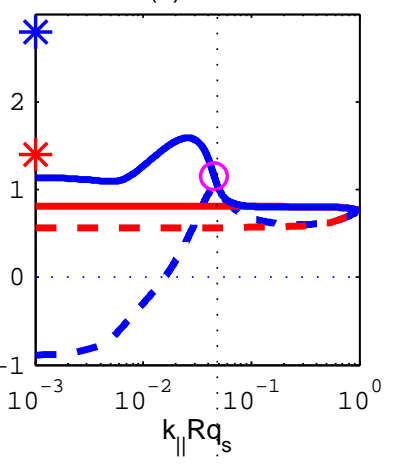

(d):

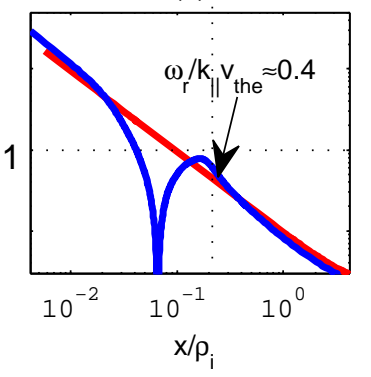

FIG. 6. Solution to the local dispersion relations Eq. (21) obtained when considering the different electron models: adiabatic (green), hybrid (red) and fully kinetic (blue), for both $(a, c)$ ITG and $(b, d)$ TEM test cases of reference described in Tab. I. In the top subplots (a) and (b), the growth rate is plotted with full lines and the real frequency with dashed lines. In the bottom subplots (c) and (d), the phase velocity normalized wrt. the electron thermal one is shown as a function of the distance $x$ to the MRS. The radial distance to the MRS $x$, in the $\mathrm{x}$-axis of (a) and (b) is mapped to the value of $k_{\|}$in the x-axis of (c) and (d) by using Eq. (13). The FWHM which corresponds to the local width estimate of the fine structure $\delta x^{\text {loc }}$ is plotted with magenta circles. Growth rates obtained with the asymptotic dispersion relation (25) are indicated with an asterisk in subplots (a) and (b).

respective positions one has $\left|\omega_{r} / k_{\|} v_{\text {the }}\right| \approx 1$ and 0.4 .

We will now systematically compare the different estimates of the fine structure widths, $\delta x^{\text {th }}, \delta x^{\text {loc }}$ and $\delta x_{\text {lin }}^{\text {sim }}$, when scanning physical parameters.

\section{Systematic scan of physical parameters and comparison of the radial width estimates}

Numerical width estimates $\delta x_{\text {lin }}^{\text {sim }}$ plotted in Fig. 8 are obtained while scanning the aforementioned physical parameters of Eq. (14): $\tau, \mu, q_{0}, \hat{s}$, and $k_{y}$. It is remarkable that a fine structure is always present over the eigenmode of the electric potential with a radial width systematically smaller than an ion Larmor radius or so, except for $\hat{s} \rightarrow 0$. It is also remarkable that the width estimate obtained from the local dispersion relation is 
(a) ITG

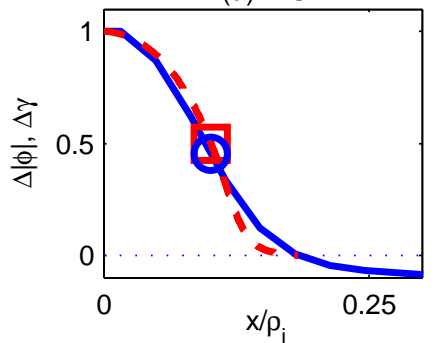

(b) TEM

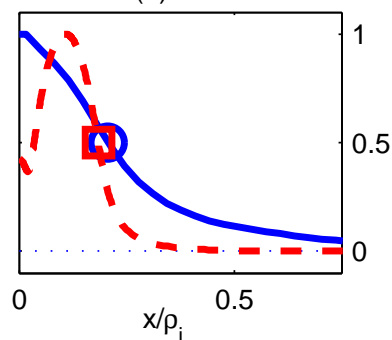

FIG. 7. Fine radial structure over the electric potential $\Delta|\phi|=\left|\widetilde{\phi}^{\text {kin }}\right|-\left|\widetilde{\phi}^{\text {hyb }}\right|$ in linear GENE simulations (blue full lines) and of the growth rate $\Delta \gamma=\left(\gamma^{\text {kin }}-\gamma^{\text {hyb }}\right)$ obtained from local dispersion relation (red dashed lines). Both curves are normalized to their maximum value as one is only interested in the radial localization of the structures. Estimates of the width $\delta x_{\text {lin }}^{\operatorname{sim}}$ and $\delta x^{\text {loc }}$ (FWHMs) are indicated with circles and squares, respectively.

in qualitative agreement with GENE simulation results, thus confirming that the radial fine structures are related to a local destabilization of the plasma due to the non-adiabatic response of passing electron near MRSs. The influence of the scanned physical parameters over the structure width can be obtained by fitting the results with power scaling laws: in the ITG case we obtain $\delta x_{\text {lin,ITG }}^{\operatorname{sim}} \propto \mu^{-1 / 2} q_{0}$ and in the TEM case we obtain $\delta x_{\text {lin,TEM }}^{\text {sim }} \propto \tau^{1 / 2} \mu^{-1 / 2} q_{0}^{3 / 2} \hat{s}^{-1} k_{y}^{-1 / 2}$. The difference of these two relations with the theoretical relation given by Eq. (14) reflects the fact that $\omega_{r}$ is not constant and its value taken from GENE varies with the scanned parameters.

In Fig. 9, for each linear simulation carried out with GENE when scanning the physical parameters, the numerical width estimate $\delta x_{\text {lin }}^{\text {sim }}$ is compared to both theoretical estimate $\delta x^{\text {th }}$ and local estimate $\delta x^{\text {loc }}$ obtained with corresponding parameters. In subplot (a), the numerical estimates obtained from GENE simulations are in good agreement with the theoretical ones, as their ratio is of order unity. In the ITG case, for the majority of scanned parameters one has $\kappa=\delta x^{\text {th }} / \delta x_{\text {lin }}^{\text {sim }} \approx 1$. In the TEM case, this velocity ratio is of the order of, but smaller, than unity: $\kappa=\delta x^{\text {th }} / \delta x_{\text {lin }}^{\operatorname{sim}} \approx 0.4$ indicating that the destabilization occurs for slower parallel wave velocities in this case than for ITG modes. It thus validates the choice of using the criteria $\left|\omega_{r} / k_{\|}\right| \approx v_{\text {the }}$ to localize, at leading order, the boundaries of the non-adiabatic region when defining the theoretical estimate $\delta x^{\text {th }}$ of the width, Eq. (14). In subplot (b), the numerical width estimates obtained from GENE $\delta x_{\text {lin }}^{\text {sim }}$ are in good agreement with the local ones $\delta x^{\text {loc }}$ for all scanned parameters of both the ITG and TEM test cases. This good agreement between the results obtained with GENE and the results obtained with the local dispersion relation, also clearly appreciable in Fig. 8, shows that the fine structures near MRSs are related to the local destabilization due to the passing electrons dynamics.

\section{FINE STRUCTURES IN NON-LINEAR SIMULATIONS AND THEIR EFFECT ON TRANSPORT}

\section{A. ITG and TEM test cases}

Non-linear simulations with hybrid and fully kinetic electrons have been carried out considering the reduced mass ratio $\mu=400$ (see table I) over a sufficiently long time (up to $t \sim 300 R / v_{t h i}$ ) to ensure good statistics over the saturated turbulent phase, as illustrated in Fig. 10 showing the time traces of the ion and electron kinetic energy fluxes, $Q_{i}$ and $Q_{e}$ respectively, for both the ITG and TEM cases. Also reported in these plots are the running time averages, $Q_{j}^{\text {run }}(t)=\int_{t_{s}}^{t} Q_{j}\left(t^{\prime}\right) d t^{\prime} /\left(t-t_{s}\right)$, taken over the turbulent saturated regime, which has been estimated to start in both cases at $t_{s}=20 R / v_{t h i}$. The final values of these running averages provide the timeaveraged fluxes over the full saturated turbulent phase of the simulation. In the following, $\langle\cdot\rangle_{t}$ stands for the time average of different physical quantities taken over the so-defined saturated time window.

Turbulence simulations involve the evolution of a spectrum of non-linearly coupled $k_{y}$ modes which consists, in our simulations, of a set of 64 modes $k_{y}=i_{y} k_{y, \text { min }}$ $\left(i_{y}=0, . ., 63\right)$, so that $k_{y, \min } \rho_{i}=0.07$ and 0.04 , in ITG and TEM cases, respectively. The $k_{x}$ spectrum consists of a set of $n_{k_{x}}=432\left(\right.$ resp. 256) modes $k_{x}=i_{x} k_{x, \text { min }}$ $\left(i_{x}=-n_{k_{x}} / 2, . ., 0, \ldots, n_{k_{x}} / 2\right)$, so that $k_{x, \min } \rho_{i}=0.032$ (resp. 0.056), in ITG (resp. TEM) case. A convergence study with respect to the number of $k_{x}$ modes, $n_{k_{x}}=n_{x}$, is conducted in Sec. IV C 2.

Shearing of turbulent eddies by turbulence-generated Zonal Flows (ZFs) is understood to be the dominant saturation mechanism of ITG-driven turbulence and therefore of the associated transport fluxes ${ }^{27-29}$. As various studies have shown ${ }^{21,30}$, the role played by ZFs in saturating TEM driven turbulence is more complex. In particular, in Ref. 30 the efficiency of turbulence saturation by ZFs is strongly dependent on the parameter range considered. It is to be noted that the physical parameters for the considered TEM case are essentially the same as the ones considered in Ref 21, except for the temperature ratio $\tau=1$ considered here instead of $\tau=3$. According to Ref 30, going from $\tau=3$ to $\tau=1$ pushes the system from a regime where ZFs play a sub-dominant role in the saturation mechanism of the TEM turbulence towards a regime where ZFs play a dominant role. This has been confirmed in our simulations by artificially zeroing out the ZFs, which led to a 10-fold increase of the turbulent fluxes, clearly reflecting the essential role played by the ZFs in regulating the turbulence.

As already mentioned when discussing the linear results, the boundary conditions require the radial length $L_{x}$ of the simulation to be an integer multiple of the distance between MRSs for each considered $k_{y}$ mode. Note 
(a)

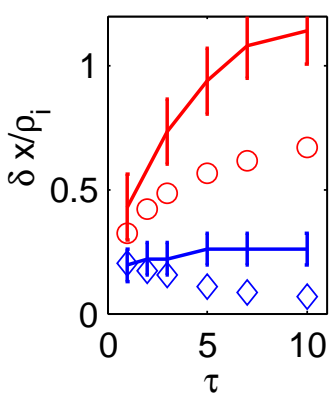

(b)

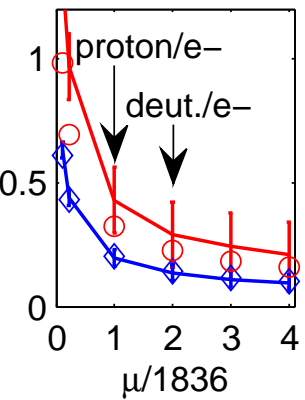

(c)

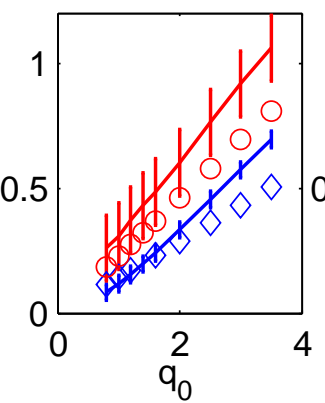

(d)

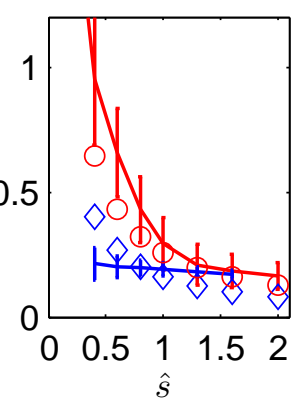

(e)

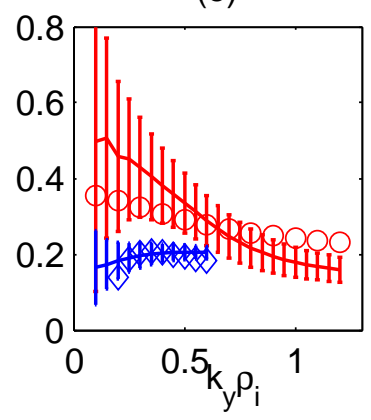

FIG. 8. Numerical estimates of the fine radial structure width with respect to different scanned parameters. Results obtained from GENE $\delta x_{\operatorname{lin}}^{\operatorname{sim}}$ are plotted in full line, with vertical error bars $\pm 2 \Delta x$ and $\Delta x=L_{x} / n_{x}$ the radial resolution, ITG in blue and TEM in red. Results obtained from the local dispersion relation $\delta x^{\text {loc }}$ : ITG in blue diamonds and TEM in red circles.
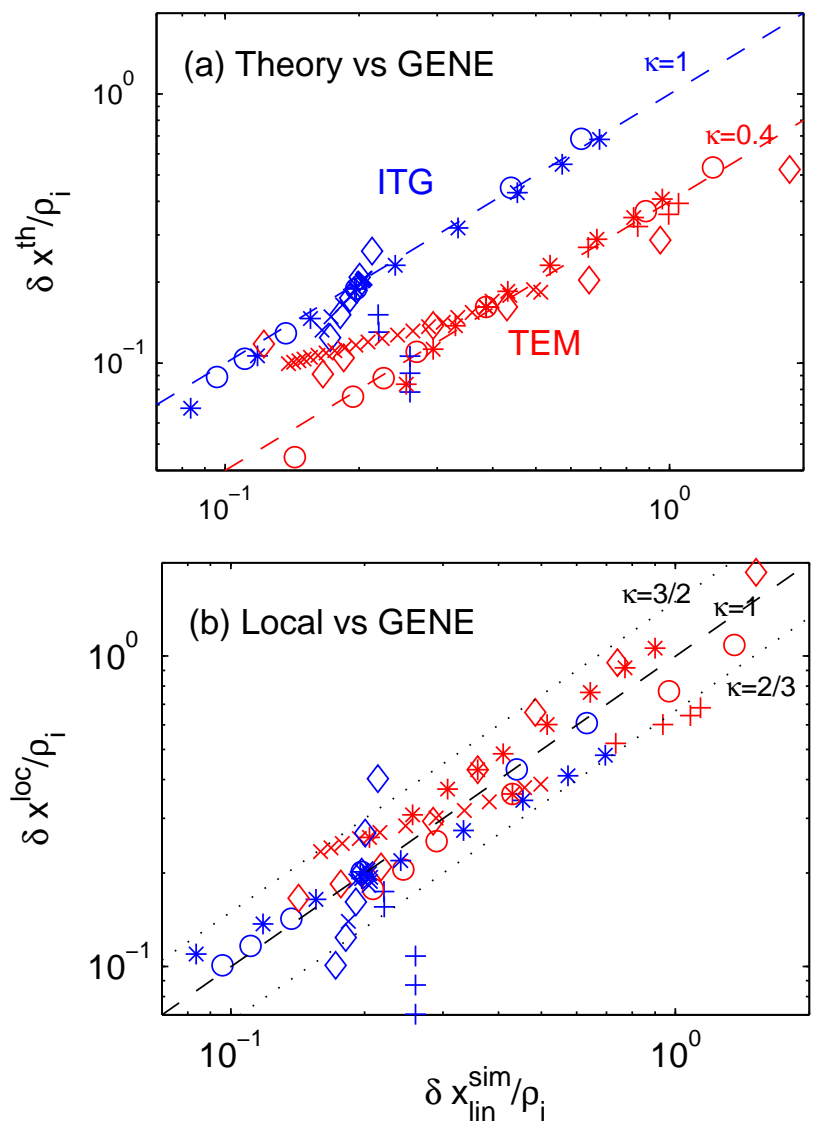

FIG. 9. Numerical width estimate $\delta x_{\text {lin }}^{\operatorname{sim}}$ of the fine structures near MRSs obtained with the FWHM in GENE versus (a) the theoretical estimate $\delta x^{\text {th }}$ using linear GENE frequencies and versus (b) the estimate $\delta x^{\text {local }}$ computed from the local dispersion relation. For the different scans of Fig. 8: $\tau(+)$, $\mu(\circ), q_{s}(*), \hat{s}(\diamond)$ and $k_{y}(\times)$. ITG in blue and TEM in red. Lines of constant slope have been plotted such that $\delta x=$ $\kappa \delta x_{\operatorname{lin}}^{\sin }$. (a) ITG
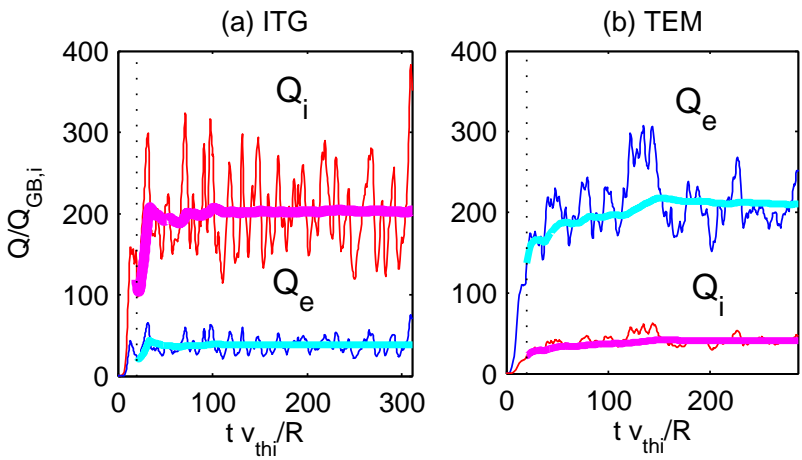

FIG. 10. (Color online) Ion and electron kinetic energy fluxes, respectively $Q_{i}$ (red) and $Q_{e}$ (blue), for both the (a) ITG and (b) TEM cases. The thicker lines are the running averages, which start at $t_{s}=20 v_{t h i} / R$ indicated with a vertical dotted line. Fluxes normalized in ion Gyro-Bohm units $Q_{\mathrm{GB}, \mathrm{i}}=$ $N_{0} T_{i 0} v_{t h i} \rho_{i}^{2} / R^{2}$.

that it is sufficient for this condition to be verified by $L_{x}$ for $k_{y, \min }$ for it to be verified for all $k_{y}$. One thus needs to set $L_{x}=M L_{\mathrm{LMRS}}$, with $M$ an integer and $L_{\mathrm{LMRS}}=1 / \hat{s} k_{y, \min }$ the distance between Lowest order MRSs. For the ITG and TEM cases, $M=8$ and 4 were chosen, giving respectively $L_{x}=142.9 \rho_{i}$ and $125.0 \rho_{i}$, thus ensuring a large enough box $\left(>100 \rho_{i}\right)$ compared to the typical radial turbulence correlation length of order $\sim 10 \rho_{i}$. The number of radial grid points $n_{x}$ is taken so as to resolve the fine structures in the corresponding linear eigenmodes of the $k_{y}$ modes which dominate the turbulent flux spectra (see Fig. 15). The values $n_{x}=432$ and 256 were thus chosen for the basic simulations, corresponding to radial grid spacings $\Delta x=L_{x} / n_{x}=0.33 \rho_{i}$ and $0.49 \rho_{i}$ for the ITG and TEM cases respectively. Figure 11 confirms that these resolutions are indeed sufficient according to this criteria. Note that the radial widths $\delta x_{\text {lin }}^{\text {sim }}$ of linear eigenmode structures as a function of $k_{y} \rho_{i}$ shown in Fig. 11 are very similar to the results shown in Fig. 8(e), these widths being however broad- 
(a) ITG

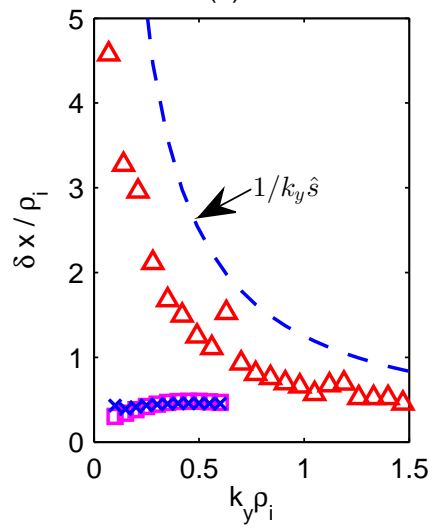

(b) TEM

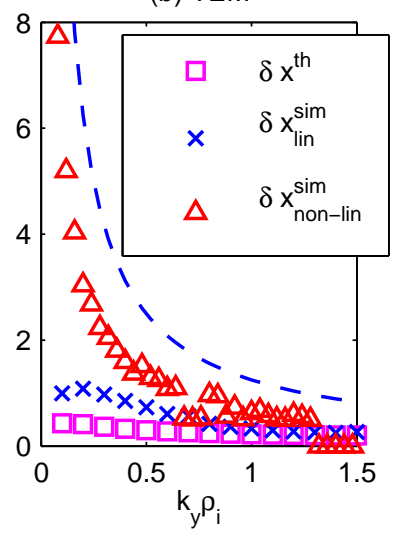

FIG. 11. Comparing fine structure widths as a function of $k_{y}$ : linear theoretical estimate $\delta x^{\text {th }}$ (Eq. (14), squares), linear GENE $\delta x_{\operatorname{lin}}^{\operatorname{sim}}(=\mathrm{FWHM}[\Delta|\widehat{\phi}|]$, crosses), and non-linear $\operatorname{GENE}\left(\delta x_{\text {non-lin }}^{\operatorname{sim}}=\mathrm{FWHM}[|\widehat{\phi}|]\right.$, triangles $)$ for both the (a) ITG and (b) TEM test cases. The distance $1 / \hat{s} k_{y}$ between MRSs is indicated with a blue dashed line. $n_{x}=432$.

ened as a result of the reduced mass ratio considered here. This is explained by the fact that $\delta x \propto \omega_{r} / \mu^{1 / 2}$ according to Eq. (14) with the real frequency $\omega_{r}$ being essentially unchanged when reducing $\mu$ from the physical value 1836 to the reduced value 400 as shown in Fig. 1. This has been confirmed with linear simulations when establishing the power scaling laws $\delta x_{\text {lin,ITG }}^{\mathrm{sim}}$ and $\delta x_{\text {lin,TEM }}^{\mathrm{sim}}$ both proportional to $\mu^{-1 / 2}$. Simulations with other values of $n_{x}$ were carried out as well and are presented in Sec. IV C 2 where the issue of radial resolution convergence of fine structures is discussed. The broadening of the radial structures are the main rationale for considering a reduced mass ratio for the non-linear simulations, as it enables to more easily ensure sufficient radial resolution with a tractable number of grid points.

Concerning the grid resolutions in the other phase space directions, $n_{z}=16$ points along $z$ were chosen for both the ITG and TEM cases. Boundaries along the velocity space directions were set to $v_{\| \text {, max }}=4.2 v_{t h}$ and $\mu_{\max }=9 \mathrm{~T} / B_{0}$ for each species both in the ITG and TEM cases, with the corresponding resolutions $n_{v_{\|}} \times$ $n_{\mu}=64 \times 8$ and $64 \times 16$ respectively. Note the slightly higher velocity resolution for the latter case, to ensure an accurate description of the trapped/passing boundary, essential for TEM simulations.

\section{B. Fine structures at MRSs}

\section{Fine structure over $k_{y} \neq 0$ modes}

The $z$ - and time- averaged amplitudes of non-zero $k_{y}$ Fourier modes obtained from the electric potential, $\left\langle\left|\widehat{\phi}_{k_{y}}\right|\right\rangle_{z t}(x, z)$, are shown in Fig. 12. These plots clearly
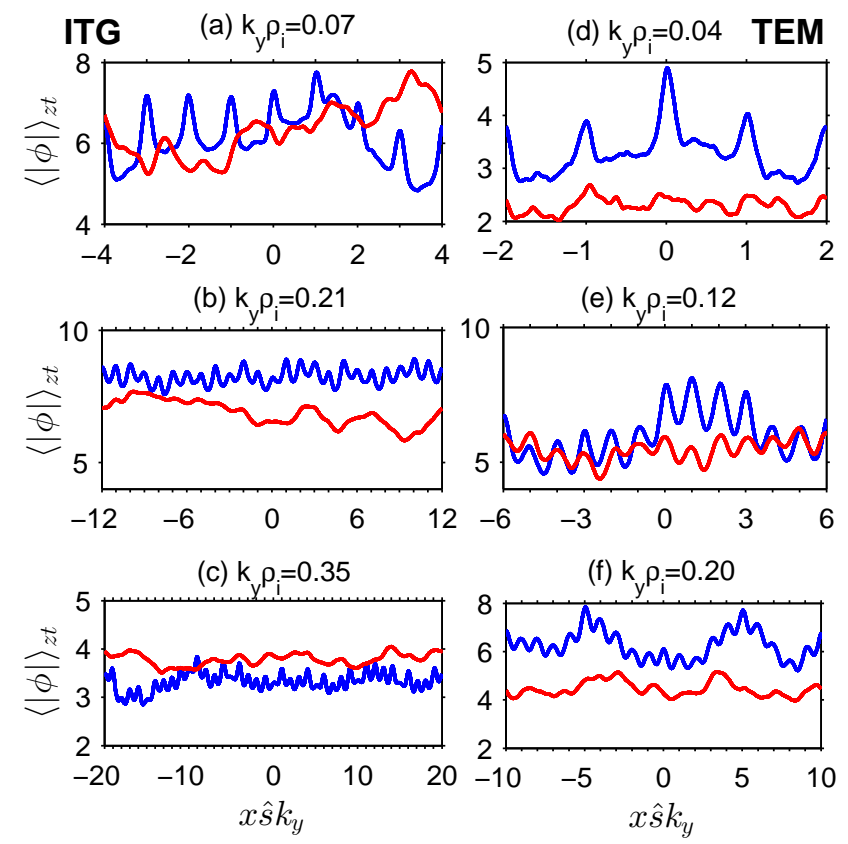

FIG. 12. Radial dependence of the $z$ - and time- averaged electric potential amplitude $\left\langle\left|\widehat{\phi}_{k_{y}}\right|\right\rangle_{z t}(x)$ for the non-linear (a-c) ITG and (d-f) TEM cases. Comparing results from both the fully kinetic (blue) and hybrid (red) electron models for different values of $k_{y} \rho_{i}$. For each $k_{y}$, associated MRSs are pointed out with tick marks along the box edge of the corresponding figure.

illustrate for each mode $k_{y} \neq 0$ the presence in the fully kinetic results of fine radial structures located at the associated MRSs, positioned at $x=\delta m / \hat{s} k_{y}, \delta m$ integer and indicated with tick marks in the corresponding plots. Similar fine structures are essentially (see the next paragraph) absent in the hybrid simulations. This confirms that the fine structures related to the non-adiabatic passing electrons and originally identified in the linear simulations actually persist in the non-linear turbulent regime. The systematic procedure applied in the linear study for measuring their radial width $\delta x$, based on subtracting the eigenmode envelope related to the hybrid computation from the one obtained with fully kinetic electrons, is however not applicable in any straightforward way for analyzing the non-linear data. One can nonetheless estimate the widths $\delta x$ by directly measuring on $\left\langle\left|\widehat{\phi}_{k_{y}}^{\text {kin }}\right|\right\rangle_{z t}(x)$ the FWHM of fine structures appearing at corresponding MRSs. As the non-linear simulation system contains multiple MRSs for each $k_{y} \neq 0$, one in fact estimates an average $\delta x$ over all associated structures. Corresponding results in Fig. 11, labeled $\delta x_{\text {non-lin }}^{\text {sim }}$, show that structures, although persisting in the non-linear regime, are significantly broadened compared to the linear widths $\delta x_{\text {lin }}^{\text {sim }}$ in both the ITG and TEM cases, by up to a factor $\sim 9$ for the lower $k_{y}$ modes.

In the TEM non-linear hybrid simulations, the particular case of the $k_{y} \rho_{i}=0.12$ mode shows, in Fig. 12 (e), 
radial corrugations over the time-averaged enveloppe of $|\phi|^{\text {hyb }}$. Indeed as shown with linear hybrid electron simulations in Fig. 2 (c) and (d), a large radial modulation of weak amplitude is already present on the linear mode enveloppe $\left|\phi^{\text {hyb }}\right|$. This weak corrugation not exactly aligned with the MRS is even more visible on the small $k_{y}$ modes in adiabatic electrons simulations $\left(k_{y} \rho_{i}<0.15\right)$ - not shown in this paper. We attribute this weak radial corrugation to the ion dynamics.

\section{Fine radial corrugation of $k_{y}=0$ modes}

In non-linear simulations, the axisymmetric Fourier modes $\left(k_{y}=0\right)$ are coupled to, and thus driven by, the unstable non-axisymmetric modes $\left(k_{y} \neq 0\right)$. As a result, the radial structures located at MRSs of $k_{y} \neq 0$ modes lead to similar structures on the $k_{y}=0$ modes in case of fully kinetic electron simulations. Such coupling is brought to the fore by studying time- and fluxsurface- averages of various fluctuation field quantities, $\langle\cdot\rangle_{F S}=\langle\cdot\rangle_{y z}$ in flux-tube coordinates. Note that averaging over the $y$-direction provides the $k_{y}=0$ Fourier component. This is clearly illustrated in Fig. 13(a-b) where the time-averaged shearing rate $\left\langle\omega_{E \times B}\right\rangle_{t}$ related to the zonal $E \times B$ flow has been plotted. This shearing rate is related to the zonal component $\langle\phi\rangle_{y z}(x, t)=\int \widehat{\phi}\left(x, k_{y}=\right.$ $0, z, t) \mathcal{J}^{\mathrm{xyz}} d z / \int \mathcal{J}^{\mathrm{xyz}} d z$ by the relation $\omega_{E \times B}(x, t) \simeq$ $\left(1 / B_{0}\right) \partial^{2}\langle\phi\rangle_{y z} / \partial x^{2}$. As will appear even clearer in Fig. 14, radial structures can be identified not only at the position of lowest order MRSs (i.e. related to $k_{y}=$ $\left.k_{y, \min }\right)$ but to the positions of next order MRSs (corresponding to $k_{y}=2 k_{y, \min }$ and $\left.k_{y}=3 k_{y, \min }\right)$ as well. This appearance of radial structures on the $k_{y}=0$ Fourier modes through non-linear coupling can be identified on essentially all fluctuation fields. As an example, the radial gradient profile $\nabla_{x}\left\langle\delta T_{e}\right\rangle_{y z t}$ of the time- and fluxsurface- averaged electron temperature fluctuation (with $\delta T_{e}$ computed using $\left.\delta T_{j}=\int d^{3} v \delta f_{j} v^{2} / 3 N_{0}-T_{j 0} \delta N / N_{j 0}\right)$ is shown in Fig. 13(c-d). Note how corresponding simulation results obtained with the hybrid electron model provide profiles with radial structures which either present much weaker amplitudes and/or whose radial positions do not appear to be correlated to MRSs. It is important to point out that in the non-linear turbulent simulations, the fine radial structures are fully revealed only after time-averaging the fluctuations. The standard deviation over time, $\sigma(x)$, of the flux-surface-averaged fluctuations have been reported in Fig. 13 as well. Note that $\sigma$ appears to be only very weakly dependent on $x$. The fact that $\sigma$ in the case of fully kinetic electron simulations is of the same order as the radial modulation amplitude of the time averages (both for $\omega_{E \times B}$ and $\left.\nabla_{x}\left\langle\delta T_{e}\right\rangle_{y z t}\right)$ reflects that the time-averaged component of the fluctuations is at least partly "drowned" by the timedependent component. This is all the more so in the case of the hybrid simulations, where the weak non-zero timeaveraged component is significantly smaller than the time dependent component [e.g. $\max _{x}\left(\left|\omega_{E \times B}\right|\right) \sim \sigma / 2$ and $\left.\max _{x}\left(\left|\nabla_{x}\left\langle\delta T_{e}\right\rangle_{y z t}\right|\right) \sim \sigma / 8\right]$.

\section{Characteristic profile modulation of flux-surface averaged fields and related gradients}

Remarkable in Fig. 13 on both radial profiles $\left\langle\omega_{E \times B}\right\rangle_{t}$ and $\nabla_{x}\left\langle\delta T_{e}\right\rangle_{y z t}$ of the fully kinetic electron simulations are their periodicity with respect to the distance $L_{\text {LMRS }}$ between lowest order MRSs. This periodicity is emphasized in Fig. 14, where the time- and flux-surfaceaveraged profiles over the full radial simulation length $L_{x}=M L_{\mathrm{LMRS}}$ of various fluctuation fields have been cut into $M$ segments which were then superimposed, the lowest order MRSs of each segment having been positioned at the center $x=0$ of these plots. The nearly perfect alignment of profile segments in the case of simulation results obtained with the fully kinetic electron model, both in the ITG and TEM case, thus clearly reflects the periodicity of radial structures, while the obvious misalignment in corresponding plots obtained with the hybrid model reflects that such periodicity of structures related to positions of MRSs is lacking or at least much weaker in these latter simulations. A hint of periodic structures aligned with the lowest order mode rational surfaces is nonetheless apparent in the hybrid electron ITG simulation, Figs. 14(b) and 14(f). These structures must however be related to the particular resonant dynamics near MRSs of passing ions and corresponding widths are therefore much wider than in the simulations with fully kinetic electrons.

In particular, the radial gradient $\nabla_{x}\langle\delta \mathcal{A}\rangle_{y z t}$ of the time- and flux-surface- averaged fluctuations for different field quantities $\mathcal{A}$ normalized with respect to corresponding background gradients $\left|\nabla_{x} \mathcal{A}_{0}\right|=\mathcal{A}_{0} / L_{\mathcal{A}}$ are plotted in the first row of Fig. 14. Shown are profiles related to density, $\mathcal{A}=N$, as well as electron and ion temperatures, i.e. $\mathcal{A}=T_{e}$ and $\mathcal{A}=T_{i}$ respectively. The appearance of non-zero profiles $\nabla_{x}\langle\delta \mathcal{A}\rangle_{y z t}$ which converge in time during the saturated turbulent phase obviously correspond to a modification of the average profiles from the initial background profiles $\mathcal{A}_{0}$. The total time- and flux-surface- averaged radial gradient profile of a given field $\mathcal{A}=\mathcal{A}_{0}+\delta \mathcal{A}$ can indeed be written:

$$
\nabla_{x}\langle\mathcal{A}\rangle_{y z t}=\nabla_{x} \mathcal{A}_{0}+\nabla_{x}\langle\delta \mathcal{A}\rangle_{y z t}=\left|\nabla_{x} \mathcal{A}_{0}\right|\left(-1+\zeta_{\mathcal{A}}\right),
$$

noting that $-\nabla_{x} \mathcal{A}_{0}=\left|\nabla_{x} \mathcal{A}_{0}\right|=\mathcal{A}_{0} / L_{\mathcal{A}}$ and having used the notation $\zeta_{\mathcal{A}}=\nabla_{x}\langle\delta \mathcal{A}\rangle_{y z t} /\left(\mathcal{A}_{0} / L_{\mathcal{A}}\right)$ for the normalized averaged fluctuation profiles as appearing in Figs. 14(a-d). Therefore where $\zeta_{\mathcal{A}}>0$, respectively $\zeta_{\mathcal{A}}<0$, the profile of $\mathcal{A}$ is locally flattened, respectively steepened, compared to the initial background $\mathcal{A}_{0}$. In particular, a value of $\zeta_{\mathcal{A}}=+1$ corresponds to a full (100\%) flattening.

One observes that in the case of fully kinetic electron simulations, for both the ITG and TEM cases, all three gradients ratios $\zeta_{N}, \zeta_{T_{e}}$, and $\zeta_{T_{i}}$ present maxima at the 
(a) ITG

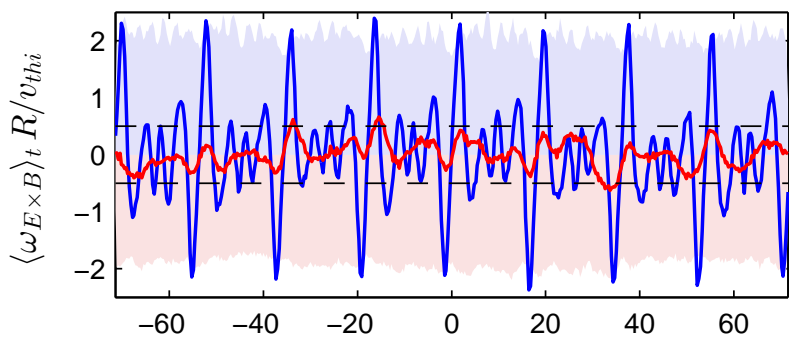

(c)

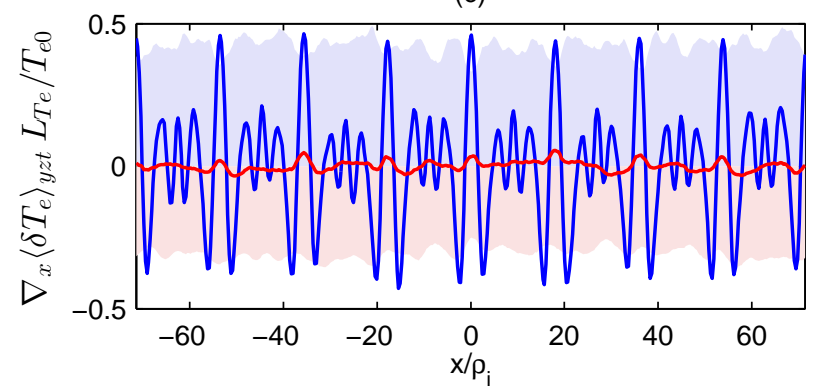

(b) TEM

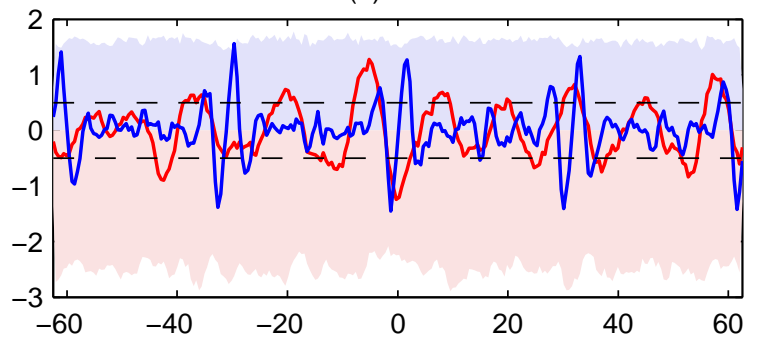

(d)

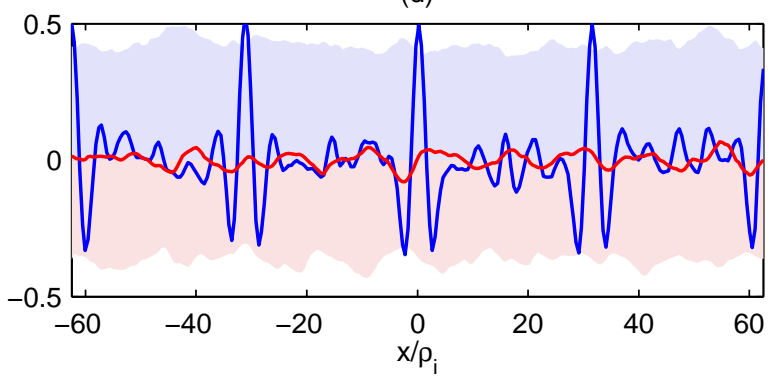

FIG. 13. (Color online) (a, b) Time-average of the $E \times B$ shearing rate $\left\langle\omega_{E \times B}\right\rangle_{t}$ related to the zonal component $\langle\phi\rangle_{y z}$, as well as $(\mathrm{c}, \mathrm{d})$ the radial gradient $\nabla_{x}\left\langle\delta T_{e}\right\rangle_{y z t}$ of the time- and flux-surface- averaged electron temperature fluctuation for hybrid (red) and kinetic (blue) electron response models. Showing results for both (a, c) the ITG and (b, d) the TEM cases. Shaded areas reflect the radially local standard deviation levels $\sigma(x)$ of corresponding fluctuation components (areas delimited by $-\sigma(x)$ and $+\sigma(x)$ for hybrid and kinetic models resp.). The maximum linear growth rate $\gamma_{\text {ref }}$, estimated as detailed in Sec. IV C 2, is plotted with horizontal dashed lines.

(a) ITG kin

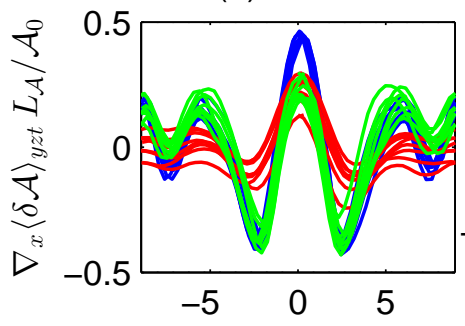

(e)

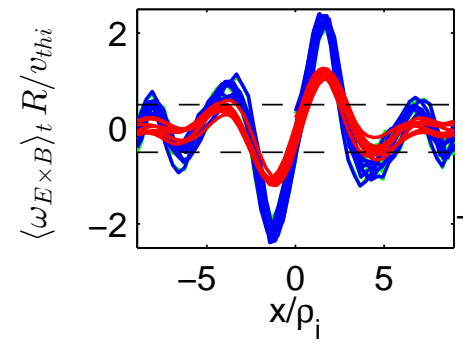

(b) ITG hyb

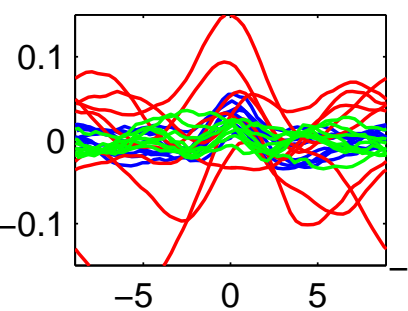

(f)

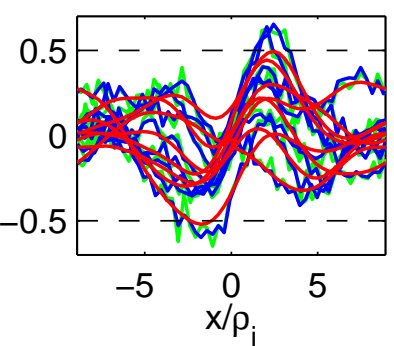

(c) TEM kin

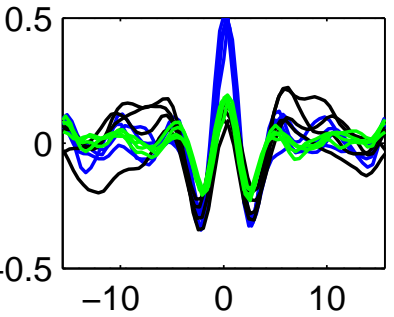

(g)

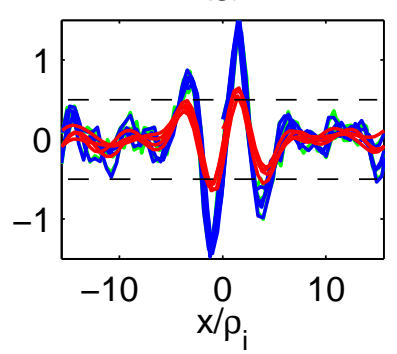

(d) TEM hyb

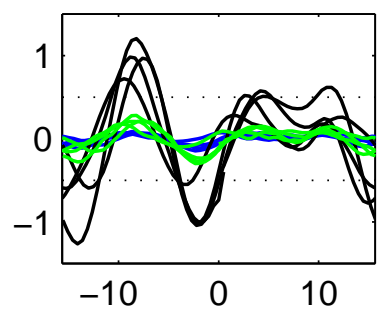

(h)

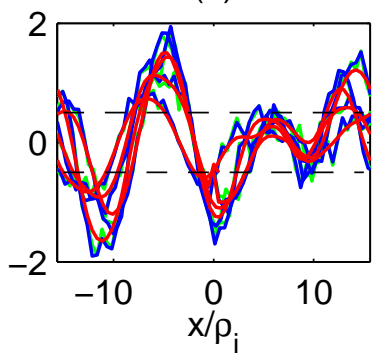

FIG. 14. (Color online). (a-d) Radial gradient $\nabla_{x}\langle\delta \mathcal{A}\rangle_{y z t}$ of the time- and flux-surface- averaged fluctuations for different field quantities $\mathcal{A}$ normalized wrt. corresponding background gradients $\left|\nabla_{x} \mathcal{A}_{0}\right|=\mathcal{A}_{0} / L_{\mathcal{A}}$ : density $\mathcal{A}=N$ (green), electron temperature $\mathcal{A}=T_{e 0}$ (blue), and ion temperature $\mathcal{A}=T_{i 0}$ (red). Profile sections of length $L_{\mathrm{LmRs}}$ have been superimposed (with lowest order MRSs at $x=0$ ), emphasizing periodicity in case of fully kinetic electron simulations. In the TEM case, $\nabla_{x} T_{i 0}=0$ so that $\nabla_{x}\left\langle\delta T_{i}\right\rangle_{y z t}$ is given in units of $T_{i 0} / \rho_{i}$ instead of $T_{i 0} / L_{T, i}$ (black). (e-f) Time-averaged shearing rate $\left\langle\omega_{E \times B}\right\rangle_{t}$ estimated with $E=-\nabla_{x}\langle\phi\rangle_{y z}$ (green) and effective shearing rate $\left\langle\omega_{E \times B}^{i, e}\right\rangle_{t}$ felt by the ions and electrons estimated with gyroaveraged $\bar{E}=-\nabla_{x}\langle\bar{\phi}\rangle_{y z}$ (red and blue respectively). Horizontal dashed lines represent $\gamma_{\mathrm{ref}}$ of linear simulations. Shown are ITG results with $(\mathrm{a}, \mathrm{e})$ kinetic and $(\mathrm{b}, \mathrm{f})$ hybrid electrons, as well as TEM results with $(\mathrm{c}, \mathrm{g})$ kinetic and $(\mathrm{d}, \mathrm{h})$ hybrid electrons. 
low order mode rational surfaces. These maxima are most prominent for the electron temperature profile at the position $x=\delta m / \hat{s} k_{y, \text { min }}$ of the lowest order mode rational surfaces, presenting values up to $\zeta_{T_{e}}=+0.5$ and thus reflecting a $50 \%$ flattening at these positions, in both the ITG and TEM cases. Somewhat lower local maximums are also clearly visible at the positions $x=\delta m / \hat{s} k_{y}$ of next order mode rational surfaces, i.e. at least for $k_{y}=2 k_{y \text {, min }}$ and $3 k_{y, \text { min }}$. Between these lowest order MRSs, $\zeta_{\mathcal{A}}$ of the different fields takes on negative values, reflecting steepening of corresponding values. Most prominent minimum values are again reached in case of the electron temperature, with values down to $\zeta_{\mathcal{A}}=-0.3$ corresponding to a $30 \%$ steepening. Due to the periodic radial boundaries in a flux-tube simulation, the radial average of the total profile gradient cannot be modified. Locally flattened regions must therefore necessarily alternate with locally steepened ones: $\left\langle\zeta_{\mathcal{A}}\right\rangle_{x}=0$.

\section{Characteristic profile modulation of $\omega_{E \times B}$ and of its gyroaverage}

In the second row of Fig. 14, the $M$ segments of the shearing rate profile $\left\langle\omega_{E \times B}\right\rangle_{t}$ have been superimposed as well. In fact, the effective electric field felt by the particles is a gyroaveraged one. As the width $\delta x$ of radial structures apparent on the $\left\langle\omega_{E \times B}\right\rangle_{t}$ shear profiles are comparable to the ion Larmor radius, an effective shearing rate $\left\langle\omega_{E \times B}^{j}\right\rangle_{t}$ should be computed separately for each species using the gyroaveraged zonal flow component of $\phi$, i.e. $\omega_{E \times B}^{j} \simeq\left(1 / B_{0}\right) \partial^{2}\langle\phi\rangle_{y z} / \partial x^{2}$, with $\bar{\phi}=\mathcal{G}_{j} \phi$ and the gyroaveraging operator $\mathcal{G}_{j}$, itself averaged over the Maxwellian background distribution, given in Fourier space by

$$
\widehat{\mathcal{G}}_{j}=\frac{B_{0}}{T_{0, j}} \int_{0}^{+\infty} d \mu \exp \left(-\frac{\mu B_{0}}{T_{0, j}}\right) J_{0}\left(\frac{k_{\perp} v_{\perp}}{\Omega_{j}}\right)=e^{-\xi_{j} / 2},
$$

recalling the notation $\xi_{j}=\left(k_{\perp} \rho_{j}\right)^{2}$. For ions, the soobtained gyroaveraged shearing rate $\left\langle\omega_{E \times B}^{i}\right\rangle_{t}$ is reduced by nearly $50 \%$ in both the ITG and TEM simulations with fully kinetic electrons. For electrons, the corresponding effective shearing rate $\left\langle\omega_{E \times B}^{e}\right\rangle_{t}$ is essentially identical to the non-gyroaveraged profile $\left\langle\omega_{E \times B}\right\rangle_{t}$. This is to be expected as the radial structures remain large with respect to electron Larmor radii. In the hybrid simulations, the structures on the $\left\langle\omega_{E \times B}\right\rangle_{t}$ profile are less affected by the gyroaveraging effects, even in case of ions, as their widths are significantly larger than in the fully kinetic electron simulations.

Finally, comparing the gradients with the $E \times B$ shearing rate, in ITG and TEM cases with kinetic electrons, it appears that $\zeta_{N}, \zeta_{T_{i}}$ and $\zeta_{T_{e}}$ have an extremum where the $\omega_{E \times B}$ shearing rate is zero. Conversely, $\zeta_{N}, \zeta_{T_{i}}$ and $\zeta_{T_{e}}$ are zero when the $\omega_{E \times B}$ is extremum. In comparison, no such regular and well defined pattern seems to appear in the hybrid simulation cases. In Fig. 13, this basic pattern organization can be clearly recognized in the
ITG case not only for the $k_{y, \text { min }}$ related MRSs but also for the $2 k_{y, \min }$ and $3 k_{y, \min }$ MRSs as already observed ${ }^{10}$ over the electron temperature profiles. The same structures related to $2 k_{y, \min }$ and $3 k_{y, \min }$ Fourier modes, although somewhat fainter, can be seen for the TEM case as well.

\section{Turbulent fluxes}

The turbulent radial fluxes result from the $x$ component of the $E \times B$ drift, given by $v_{E \times B, x}=$ $-\left(1 / B_{0}^{2}\right)\left(\nabla \phi \times \mathbf{B}_{0}\right) \cdot \nabla x /|\nabla x| \simeq-\left(1 / B_{0}\right) \partial \phi / \partial y$, where $\phi$ is the scalar potential related to the essentially electrostatic fluctuations. One notes that although magnetic fluctuations have been accounted for in the fully kinetic simulations, their contribution to the turbulent fluxes represent less than $1 \%$ of the total fluxes and are not discussed here. The electrostatic fluxes, specific to each species $j$ and averaged over the flux-tube volume $V=\int d^{3} x=\int d x d y d z \mathcal{J}^{\mathrm{xyz}}$, are essentially of the form:

$$
\mathcal{F}_{j}[\bullet](t)=\frac{1}{V} \int d^{3} x \frac{1}{B_{0}}\left(-\frac{\partial \phi}{\partial y}\right)(\mathbf{x}, t) \int d^{3} v \bullet \delta f_{j}(\mathbf{x}, \mathbf{v}, t) .
$$

In particular, the particle flux and kinetic energy flux are given by $\Gamma_{j}=\mathcal{F}_{j}[1]$ and $Q_{j}=\mathcal{F}_{j}\left[m_{j} v^{2} / 2\right]$, and have been normalized to the following ion Gyro-Bohm units $\Gamma_{G B, i}=N_{0} v_{t h i} \rho_{i}^{2} / R^{2}$ and $Q_{G B, i}=N_{0} T_{i 0} v_{t h i} \rho_{i}^{2} / R^{2}$, respectively. In our ITG and TEM reference cases $Z=1$ and therefore $\Gamma_{i}=\Gamma_{e}=\Gamma$.

A fundamental issue, due to limited simulation statistics, is in providing error estimates of time-averaged fluxes $\langle\mathcal{F}\rangle_{t}$. The practical approach considered here consists in dividing the quasi-stationary turbulent phase of the simulation into $\mathrm{N}$ disjoint time intervals of equal span and computing the time averages $\langle\mathcal{F}\rangle_{n}$ over each of these sub-intervals. An estimate of the error $\left\langle v^{2}\right\rangle_{t}$ on the total time-average flux can then be provided by the root mean square deviation:

$$
\operatorname{Err}[\mathcal{F}]=\left[\frac{1}{N} \sum_{n=1}^{N}\left(\langle\mathcal{F}\rangle_{n}-\langle\mathcal{F}\rangle_{t}\right)^{2}\right]^{1 / 2}
$$

The number of time intervals $N$ one can consider is usually very limited (typically 3 for our simulations) as each must contain at least a few $(\sim 10)$ turbulent bursts. The rough error estimate on the time-averaged fluxes obtained in this way is less than $5 \%$ for non-linear simulation results shown in Figs. 15, 16, and 17.

\section{1. $\quad k_{x}$ and $k_{y}$ Spectra}

Flux spectra enable to study the contribution to transport from the different fluctuation scale lengths. Such spectra are derived by expressing relation (26) in terms of the Fourier representations of $\phi$ and $\delta f_{j}$ with respect 

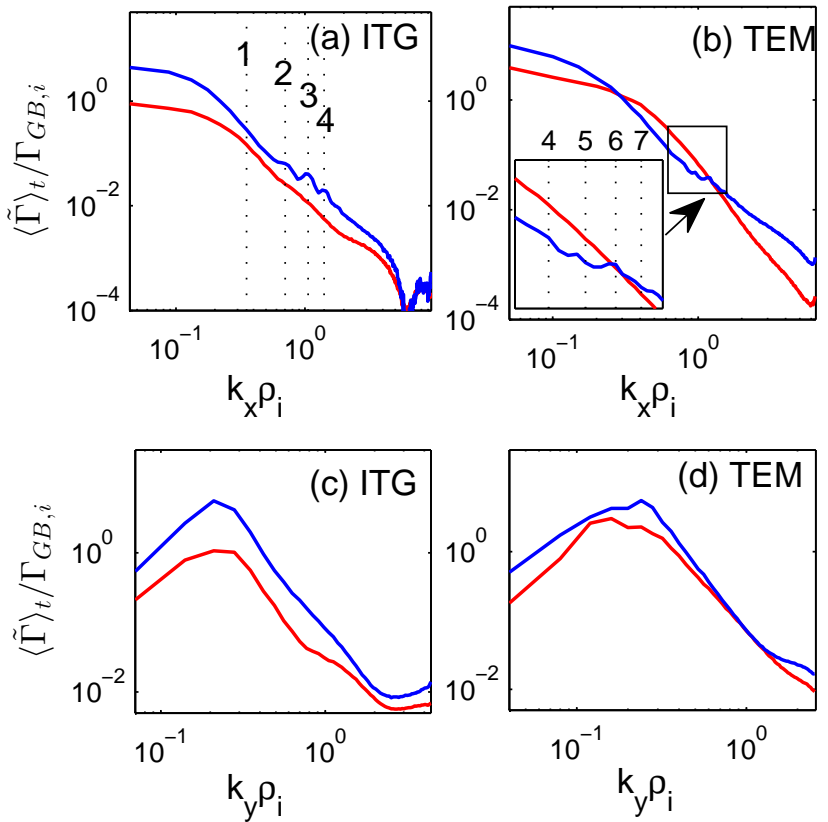

FIG. 15. Time-averaged, turbulent particle flux spectra $\langle\widetilde{\Gamma}\rangle_{t}$ in units of $\Gamma_{G B, i}$ with respect to (a and b) $k_{x}$ and (c and d) $k_{y}$ for the ITG (a and c, $n_{x}=432$ ) and TEM (b and d, $n_{x}=256$ ) test cases (log-log scale). Fully kinetic (blue) and hybrid (red) electron models. Peaks in the fully kinetic electron spectra at the harmonics $k_{x}=p 2 \pi / L_{\mathrm{LMRS}}, p=1,2, \ldots$, are related to the non-adiabatic response of passing electrons near lowest order MRSs.

to $x$ and $y$. In particular, the Fourier decomposition of $\phi$ reads:

$$
\phi(x, y, z, t)=\sum_{k_{x}, k_{y}} \widehat{\phi}\left(k_{x}, k_{y}, z, t\right) \exp \left[\imath\left(k_{x} x+k_{y} y\right)\right] .
$$

In the case of particle flux, for example, equation (26) then becomes:

$\Gamma_{j}(t)=\sum_{k_{x}, k_{y}} \frac{\int d z \mathcal{J}^{\mathrm{xyz}} B_{0}^{-1} i k_{y} \widehat{\phi}^{\star} \widehat{\delta N}_{j}}{\int d z \mathcal{J}^{\mathrm{xyz}}}=\sum_{k_{x}, k_{y}} \widetilde{\Gamma}_{j}\left(k_{x}, k_{y}, t\right)$,

having identified the flux spectra as

$$
\widetilde{\Gamma}_{j}\left(k_{x}, k_{y}, t\right)=\left\langle\frac{1}{B_{0}} \imath k_{y} \widehat{\phi}^{\star} \widehat{\delta N}_{j}\right\rangle_{z}
$$

where $\widehat{\phi}^{\star}$ is the complex conjugate of $\widehat{\phi}$.

The time-averaged $k_{x^{-}}$and $k_{y^{-}}$particle flux spectra are plotted for both the ITG and TEM cases in Fig. 15, obtained by summing $\langle\widetilde{\Gamma}\rangle_{t}\left(k_{x}, k_{y}\right)$ over $k_{y}$ and $k_{x}$, respectively. For comparison, the figure shows simulation results obtained with both the fully kinetic and hybrid electron models. All these spectra present a typical decay towards the shorter wavelengths, which is roughly algebraic, $\sim k^{-\alpha}$, corresponding to a straight line in log$\log$ scale, often referred to as the "inertial range". The contributions to the fluxes from the shortest considered wavelength modes are thus at least three orders of magnitude smaller than the one from the spectrum peak, typically located at $k_{y} \rho_{i}=0.2-0.3$, which is a clear indication of a well-converged turbulent simulation in terms of the number of Fourier modes considered. The spectrum peak itself is well resolved, reflecting a good spectral resolution $\Delta k_{x, y}=k_{x, y, \min }=2 \pi / L_{x, y}$, i.e. a sufficiently large simulation box. In the ITG case, at the very edge (Nyquist limit) of the spectra, one observes however a slight deviation from the algebraic decay, in the form of a flattening or even roll-over for the $k_{y}$-spectra. This is clearly not due to a linearly unstable mode at these scales, as shown in Fig. 1(a) (see results for $\mu=400$ and $\tau=1$ ), but is most probably related to a pile-up of energy cascading down toward small scales. In the TEM case, no roll-over is present at the very edge (Nyquist limit) of the spectra even if the curve's decay slightly lessen for $k_{y} \rho_{i} \approx 1.5$. The absence of roll-over has also been confirmed for simulations with higher radial resolutions.

Remarkable on the $k_{x}$-spectra of fully kinetic electron simulations are peaks at harmonics $k_{x}^{(p)}=p 2 \pi / L_{\mathrm{LMRS}}=$ $p M k_{x, \min }$. These peaks are most clearly visible in the particle flux spectra of the ITG case, and most prominent for $p=2,3$, and 4 . These are clearly related to the Fourier mode components giving rise to the periodic structures located at the lowest order MRSs and are thus absent from the hybrid simulation results. The origin of the peaks in the time-averaged $k_{x}$ spectra $\langle\widetilde{\Gamma}\rangle_{t, k_{y}}\left(k_{x}\right)=\sum_{k_{y}}\langle\widetilde{\Gamma}\rangle_{t}\left(k_{x}, k_{y}\right)$ is shown to be due to the presence of peaks at aligned along $\left(k_{y}=k_{x} / 2 \pi \hat{s}\right)$ in the time-averaged bi-dimensional spectra $\langle\widetilde{\Gamma}\rangle_{t}\left(k_{x}, k_{y}\right)$ which are plotted in Fig. 16. These modes correspond, for each $k_{y}$, to the first order coupling to the mode $\left(k_{x}, k_{y}\right)=\left(0, k_{y}\right)$ resulting from the pseudo periodicity boundary conditions described by Eq. (1). Very similar features are also observed on both electron and ion kinetic energy flux spectra (not shown).

\section{Numerical radial resolution}

As can be observed in Fig. 11, the widths of the fine structures roughly scale as half the distance between consecutive lowest order MRSs for each $k_{y}$. Non-linear simulation cases considered in this work are composed of 64 $k_{y}$ modes; the biggest $k_{y}$ mode has the highest number of MRSs $(64 \times M)$ within the box boundaries: $512($ ITG) and 256 (TEM). As a result, if one wants for example a minimum of 10 points between the MRSs to resolve the fine radial structures of all modes, it respectively requires 5120 and 2560 points in $x$. Nonetheless, as discussed in Sec. IV C 1, the turbulence spectra is dominated by low $k_{y}$ modes $\left(k_{y} \rho_{i}<0.3\right)$ which have the largest radial structures at MRSs: $\delta x_{\text {non-lin }}^{\text {num }} \gtrsim 2 \rho_{i}$. To have at least 10 points between these $k_{y}$ 's lowest order MRSs, it requires to have $n_{x}>340$ and 300 . In our convergence 
(a) ITG Kin

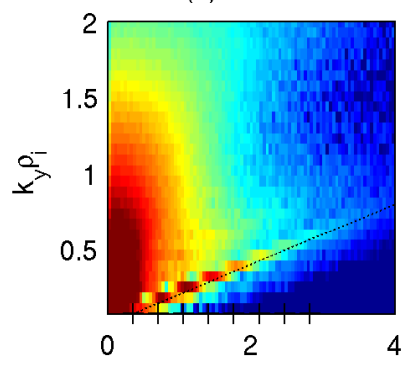

(c) ITG hyb

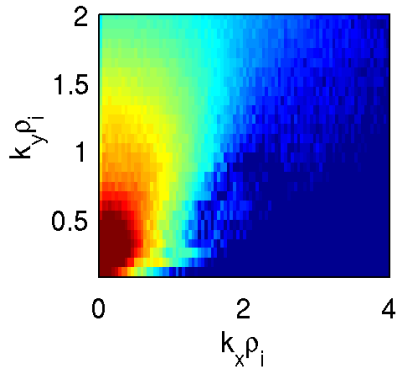

(b) TEM kin

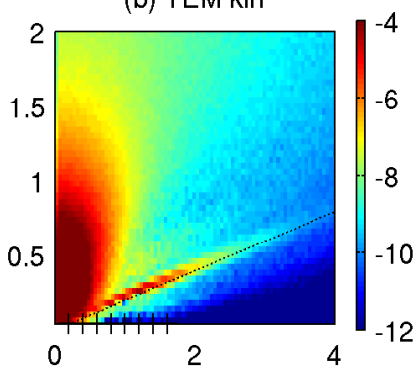

(d) TEM hyb

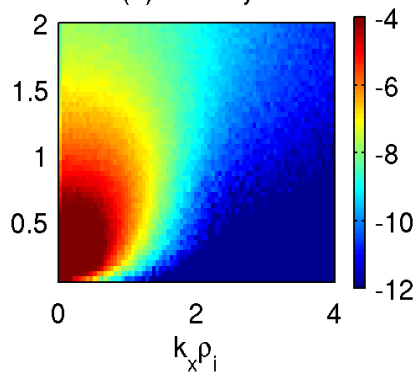

FIG. 16. Time-averaged, turbulent particle flux spectrum $\langle\widetilde{\Gamma}\rangle_{t}\left(k_{x}, k_{y}\right)$ for same cases as in Fig. 15 (shown is $\left.\log \langle\widetilde{\Gamma}\rangle_{t}\left(k_{x}, k_{y}\right)\right)$. ITG (a and b) and TEM (c and d) cases with kinetic $(\mathrm{a}, \mathrm{b})$ and hybrid $(\mathrm{c}, \mathrm{d})$ electron models. For the kinetic results, a black dotted line is plotted at $k_{y}=k_{x} / 2 \pi \hat{s}$ to emphasize the $\left(k_{x}, k_{y}\right)$ coupling due to pseudo-periodic boundary conditions Eq. (1) and positions of the harmonics $k_{x}^{(p)}$ with $p=1 . .8$ are plotted with black tick marks along the $\mathrm{x}$-axis. The $k_{y}=0$ modes are not shown as they do not contribute to the transport in the $\nabla x$ direction: $\partial / \partial y \equiv-\imath k_{y}=0$.

study, we went beyond this resolution by taking $n_{x}=512$ and 1024 . Note that these two runs with very high radial resolutions have been carried out over a shorter time window $t_{\text {end }} \approx 200 v_{\text {thi }} / R$ compared to other simulations for which $t_{\text {end }} \approx 300 v_{\text {thi }} / R$.

In the convergence study (Fig. 17), we scanned a range of radial point numbers, $n_{x}$, for which the mesh size in units of the ion Larmor radius, $\Delta x / \rho_{i}=L_{x} / n_{x} \rho_{i}$, varies from 0.28 to 1.49 in the ITG case and from 0.12 to 1.30 in the TEM case. The highest resolutions provide more than 7 (ITG) and 16 (TEM) radial grid points over the fine structure widths, $\delta x_{\text {non-lin }}^{\text {sim }}$ of dominant $k_{y}$ modes $\left(k_{y} \rho_{i}<0.3\right)$. In comparison, the lowest resolutions provide less than two grid points over the fine structure widths of almost all $k_{y}$ modes. This low resolution is clearly not sufficient to describe the radial corrugations present near MRSs in kinetic electron simulations. In Fig. 17, we show with dashed lines the linear fits that have been extrapolated to obtain the converged values $(\Delta x \rightarrow 0)$.

The particle and kinetic energy fluxes of ions and electrons, as well as the effective $E \times B$ shearing rate $\omega_{\text {eff }}$, which are obtained from simulations running with the hybrid model are essentially converged already for low res-
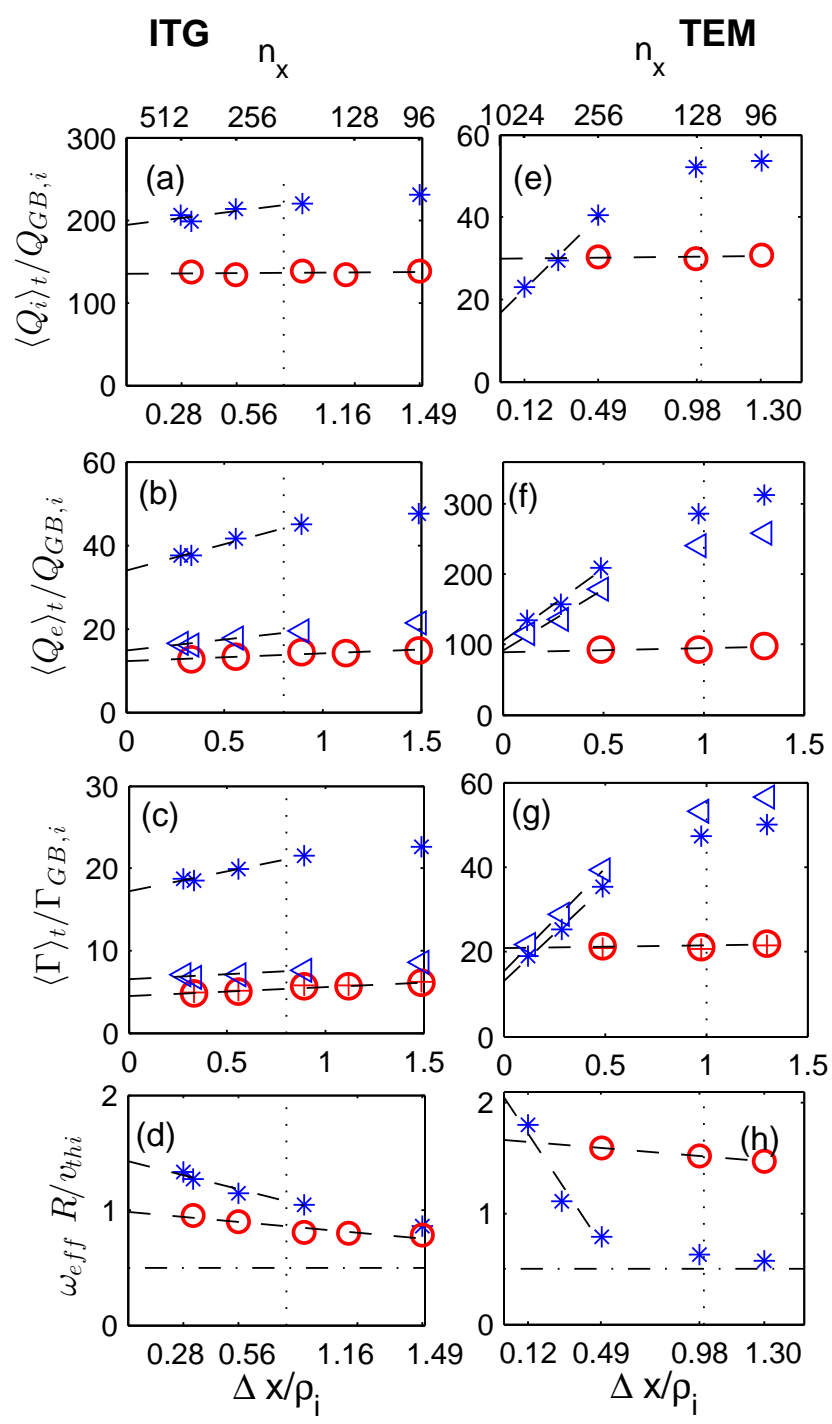

FIG. 17. Flux-surface and time averaged particle and kinetic energy fluxes plotted with respect to the radial resolution $\Delta x=L_{x} / n_{x} \rho_{i}$, and effective $E \times B$ shearing rate $\omega_{\text {eff }}\left(t_{w}=\gamma_{\text {ref }}^{-1}\right)$ plotted with respect to the number of points in the radial direction $n_{x}$. In ITG $(\mathrm{a}, \mathrm{b}, \mathrm{c}, \mathrm{d})$ and TEM $(\mathrm{e}, \mathrm{f}, \mathrm{g}, \mathrm{h})$ regimes with hybrid (circles) and kinetic (asterisk) electrons. Also plotted are the fluxes solely due to the trapped electrons (triangle). In hybrid simulations particle flux is computed with ion (circles) and with trapped electrons (crosses). $\Gamma_{G B, i}=N_{0} v_{t h i} \rho_{i}^{2} / R^{2} ; Q_{G B, i}=N_{0} T_{i 0} v_{t h i} \rho_{i}^{2} / R^{2}$. Vertical dotted lines indicate the fine structure width of $k_{y} \rho_{i} \leq 1$ modes in linear simulations taken from Fig. 11.

olutions (circles in Fig. 17). On the contrary, the fluxes and $\omega_{\text {eff }}$ obtained from simulations carried out with the kinetic electron model (asterisk in Fig. 17) are not converged at low resolutions. In subplot (h), the $\omega_{\text {eff }}$ does not appear clearly converged, even at the highest resolution. As we discuss now, the effective shearing rate $\omega_{\text {eff }}$ considered in this work includes a fluctuating contribution. The following results show that the strong increase 
of the effective shearing rate, occurring in the TEM case when $\Delta x \rightarrow 0$, is due to the contribution from the rapid fluctuations. The effective $E \times B$ shearing rate is defined by:

$$
\omega_{e f f}\left(t_{w}\right)=\left\langle\left|\frac{1}{t_{w}} \int_{t}^{t+t_{w}} d t^{\prime} \omega_{E \times B}\left(x, t^{\prime}\right)\right|\right\rangle_{x t},
$$

which is a function of the time-window $t_{w}$. In the last row of Fig. 17, the effective shearing rate is plotted using a value of the time window equal to the the typical growth time of the turbulence: $t_{w}=\gamma_{r e f}^{-1}$. This choice permits to filter out the $\omega_{E \times B}$ fluctuations assumed to oscillate too rapidly to be able to suppress turbulence ${ }^{31,32}$ but to still account for the slower ones which should be effective in suppressing turbulence. In the ITG case, this particular time window is chosen as the inverse of the maximum linear growth rate of the dispersion relation in Fig. 1(a). In the TEM case, it is chosen as the maximum growth rate over the spectrum interval $0.1<k_{y} \rho_{i}<0.4$ of the dispersion relation in Fig. 1(b). This limited spectrum interval considered for the TEM case corresponds to modes which contribute the most to the turbulent fluxes (see 15(d)).

The fact that the $\omega_{\text {eff }}$ is essentially converged at lower resolution in hybrid electron simulations is clearer in Fig. 18 (dashed lines). In this figure, the effective shearing rate is plotted with respect to the time window width for different radial resolutions. In the TEM case (Fig. 18.b) with kinetic electrons (full line), the effective shearing rate strongly increases when the mesh size $n_{x}$ increases, especially for small values of the time window $t_{w}$. This short scale effect is particularly important in the highest resolution simulation. Going back to Fig. 17 (f) and $(\mathrm{h})$, we note that going to higher resolution has the effect of increasing $\omega_{\text {eff }}$ while decreasing the turbulent fluxes. This is in agreement with the drift wave-zonal flow paradigm for non-linear saturation. Comparing the $\omega_{\text {eff }}$ between hybrid and kinetic simulations is not forcefully significant as the difference of the ZFs spatial organization is not taken into account by this indicator; but comparing the $\omega_{\text {eff }}$ between simulations with the same electron model and the same magnetic equilibrium, i.e. the same spatial organization of ZFs, one can expect that with a larger $\omega_{\text {eff }}$ the ZFs will be more effective for suppressing turbulence.

As a conclusion of this section, we emphasize the necessity to use a high radial resolution for converging the flux levels and associated $E \times B$ saturation mechanisms. Fully kinetic simulations carried out with too low radial resolution can lead to strong over-estimate of the fluxes correlated to a strong under-estimate of the $E \times B$ shearing rate.

\section{Passing electrons contributions to the turbulent transport}

In this section, we note $\langle\bullet\rangle_{t}$ the time averaged level of transport extrapolated at $\Delta x=0$ by linear fits indicated with dashed lines in Fig. 17.

a. In the ITG case, the particle and kinetic energy fluxes of ion and electron species are underestimated when running simulations using the hybrid electron model: $\left\langle\Gamma_{e}^{\mathrm{hyb}}\right\rangle_{t} \approx 27 \%\left\langle\Gamma_{e}^{\mathrm{kin}}\right\rangle_{t},\left\langle Q_{e}^{\mathrm{hyb}}\right\rangle_{t} \approx 36 \%\left\langle Q_{e}^{\mathrm{kin}}\right\rangle_{t}$, and $\left\langle Q_{i}^{\text {hyb }}\right\rangle_{t} \approx 69 \%\left\langle Q_{i}^{\text {kin }}\right\rangle_{t}$. The error due to the extrapolation method is small enough to be confident in these numbers.

The particle flux is composed of a passing electron flux and of a trapped electron flux: $\Gamma_{e}=\Gamma_{e, p a s}+\Gamma_{e, t r p}$. In simulations running with kinetic electrons, the main contribution to the flux comes from the passing electron channel of transport: $\left\langle\Gamma_{e, p a s}^{\mathrm{kin}}\right\rangle_{t} \approx 63 \%\left\langle\Gamma_{e}^{\mathrm{kin}}\right\rangle_{t}$. The passing electrons being adiabatic in the hybrid model, one obviously has $\Gamma_{e, p a s}^{\text {hyb }}=0$. The trapped electron particle fluxes obtained from simulations running with hybrid or kinetic electron models are of same order: $\Gamma_{e, t r p}^{\mathrm{hyb}} \approx \Gamma_{e, t r p}^{\mathrm{kin}}$

The kinetic energy flux is composed of a heat flux ${ }^{33}$ and of an advective flux:

$$
Q_{j}=q_{\mathrm{h}, j}+\frac{5}{2} T_{0 j} \Gamma_{j}
$$

where the heat flux ${ }^{33}$ is defined by $q_{\mathrm{h}, j}=\mathcal{F}_{j}\left[m_{j}\left(v^{2}-\right.\right.$ $\left.\left.5 v_{t h j}^{2}\right) / 2\right]$ and $\mathcal{F}_{j}[\bullet]$ is defined by Eq. (26). With the fully kinetic model, the advective contribution from the passing electrons dominates the electron kinetic energy flux: $(5 / 2) T_{e 0}\left\langle\Gamma_{e, p a s}^{\mathrm{kin}}\right\rangle_{t} \approx 78 \%\left\langle Q_{e}^{\mathrm{kin}}\right\rangle_{t}$.

The ion kinetic energy flux, which is the most important for an ITG regime, is underestimated by simulations running with the hybrid model: $\left\langle Q_{i}^{\text {hyb }}\right\rangle_{t} \approx 69 \%\left\langle Q_{i}^{\text {kin }}\right\rangle_{t}$. Having ambipolar fluxes, $\left\langle\Gamma_{i}\right\rangle_{t}=\left\langle\Gamma_{e}\right\rangle_{t}$, the hybrid model underestimation of the kinetic energy flux, i.e. $\left\langle Q_{i}^{\text {hyb }}\right\rangle_{t}<$ $\left\langle Q_{i}^{\text {kin }}\right\rangle_{t}$, is significantly due to the missing passing electron channel of transport: $(5 / 2) T_{e 0}\left\langle\Gamma_{e, p a s}^{\text {hyb }}\right\rangle_{t}=0$.

$b$. In the TEM case, the extrapolated levels of transport obtained with the hybrid and kinetic models are: $\left\langle\Gamma_{e}^{\mathrm{hyb}}\right\rangle_{t} \approx 61 \%\left\langle\Gamma_{e}^{\mathrm{kin}}\right\rangle_{t},\left\langle Q_{e}^{\mathrm{hyb}}\right\rangle_{t} \approx 85 \%\left\langle Q_{e}^{\mathrm{kin}}\right\rangle_{t}$, and $\left\langle Q_{i}^{\text {hyb }}\right\rangle_{t} \approx 180 \%\left\langle Q_{i}^{\text {kin }}\right\rangle_{t}$. In absolute value, the differences between these hybrid and kinetic fluxes are of the order of the uncertainty on their amplitude due to the choice of extrapolation method.

The particle flux, in simulation with kinetic electrons, is mainly composed of the trapped electrons transport channel: $\left\langle\Gamma_{e, t r p}^{\mathrm{kin}}\right\rangle_{t} \approx 119 \%\left\langle\Gamma_{e}^{\mathrm{kin}}\right\rangle_{t}$, and the passing electron flux is inward. The trapped electron fluxes obtained from simulations carried out with hybrid and kinetic electron models are of comparable level: $\left\langle\Gamma_{e, t r p}^{\text {hyb }}\right\rangle_{t} \approx$ $134 \%\left\langle\Gamma_{e, t r p}^{\mathrm{kin}}\right\rangle_{t}$. The difference being of the order of the error due to the extrapolation method.

The electron kinetic energy flux is the major transport channel causing power loss in this TEM case, as expected of a TEM regime. In simulation with kinetic electrons, this flux is essentially due the trapped electrons channel: $\left\langle Q_{e, t r p}^{\mathrm{kin}}\right\rangle_{t} \approx 87 \%\left\langle Q_{e}^{\mathrm{kin}}\right\rangle_{t}$, and it has a non negligible advective component (see Eq. (29)): $(5 / 2) T_{e 0}\left\langle\Gamma_{e}^{\mathrm{kin}}\right\rangle_{t} \approx 37 \%\left\langle Q_{e}^{\mathrm{kin}}\right\rangle_{t}$. Not shown here is the fact that, in both hybrid and kinetic simulations of the TEM 

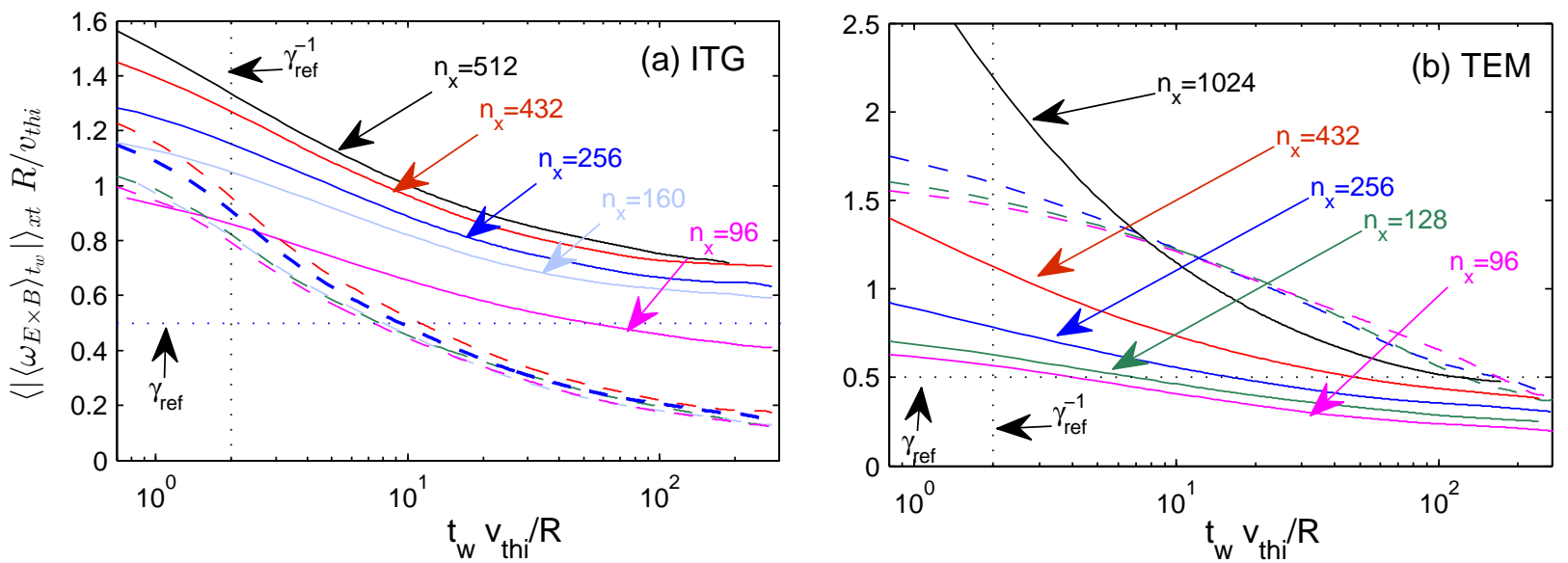

FIG. 18. Effective shearing rate $\omega_{e f f}=\left\langle\left|\left\langle\omega_{E \times B}\right\rangle_{t_{w}}\right|\right\rangle_{x t}$ with respect to a time window $t_{w}$ for different radial resolutions indicated for (a) ITG and (b) TEM test cases. Both hybrid (dashed) and kinetic (full line) electron models are considered here where each resolution respects the same color code as indicated in the plots. The linear growth rate of reference $\gamma_{\text {ref }}$ is plotted with horizontal dotted line and its corresponding time $\gamma_{\text {ref }}^{-1}$ is plotted with vertical dotted line.

case, the electron heat flux is solely due to the trapped electrons: $\left\langle q_{\mathrm{h}, e}^{\text {kin }}\right\rangle_{t}=\left\langle q_{\mathrm{h}, e, t r p}^{\text {kin }}\right\rangle_{t}$ and $\left\langle q_{\mathrm{h}, e}^{\text {hyb }}\right\rangle_{t}=\left\langle q_{\mathrm{h}, e, t r p}^{\text {hyb }}\right\rangle_{t}$. The transport levels obtained from hybrid and kinetic electron simulations are only different by $\approx 15 \%$. This is not a significant difference regarding the error due to the extrapolation method.

The transport levels of ion kinetic energy obtained with the kinetic and hybrid electron models are different enough to at least say that this flux is decreased by the influence of the passing electron dynamics. But this ion kinetic energy flux remains much lower than the electron kinetic energy flux.

\section{CONCLUSION}

The non-adiabatic response of passing electrons near mode rational surfaces has been characterized in the flux-tube geometry by identifying its role in linear and non-linear collisionless plasma, studying instabilities and turbulent saturated regimes of an ITG and a TEM test cases. This characterization was achieved by systematically comparing results obtained when accounting for the fully kinetic response of the electrons with results obtained with an hybrid model in which the trapped electrons are handled kinetically and the passing electrons are forced to respond adiabatically.

In linear simulations, fine radial structures due to the non-adiabatic response of passing electrons are systematically present near MRSs. These fine structures develop in the vicinity of MRSs where $k_{\|}$is small enough to have $\left|\omega_{r} / k_{\|}\right| \gtrsim v_{\text {the }}$. The condition $\left|\omega_{r} / k_{\|}\right|=v_{\text {the }}$ appears to be a good criteria to localize the boundaries of the region where passing electrons are non-adiabatic. The dependence on the main physical parameters of the fine structure radial width has been characterized.
For comparison, the underlying destabilization occurring near MRS and related to the passing electrons dynamics have been described with a local dispersion relation. It was shown that in the radial region where the fine structure related to the non-adiabatic response of passing electrons is present, the growth rate obtained with the local dispersion relation is significantly raised, by a factor two or more, when including the non-adiabatic response of passing electrons. The radial width estimate based on this local growth rate was found to be in good agreement with the one directly measured in the results from GENE numerical simulations.

This local dispersion relation has then been reduced to a second order polynomial equation, in order to compute an analytical solution. In this simple form, the ion finite Larmor radius effects cannot be neglected if one wants to correctly describe the destabilization mechanism due to the passing electrons near MRS. Finally, with this analytical dispersion relation, an instability condition showing the interchange nature of the instability at MRS has been found for both ITG and TEM cases.

In non-linear simulations, fine structures due to the non-adiabatic response of passing electrons over $k_{y} \neq 0$ modes persist in the turbulent saturated regime, somewhat broadened compared to the linear case. By nonlinear coupling with the $k_{y}=0$ mode, it leads to periodic radial corrugations over the flux-surface- and timeaveraged gradients of temperature and density, as well as over the time-averaged $E \times B$ shearing rate. Consequently, dominant $k_{y}$ modes which contribute the most to the fluxes, as illustrated in direct space and Fourier space, require to be finely resolved in the radial direction to properly account for the particular dynamics of passing electrons near MRSs. An effective $E \times B$ shearing rate was introduced which filters out the (too) rapidly oscillating component of the $\omega_{E \times B}$.

It has been necessary, with the fully kinetic model, to 
use a much higher radial resolution for converging the flux levels and associated $E \times B$ saturation mechanisms than with the hybrid model. Fully kinetic simulations carried out with too low radial resolution can lead to strong over-estimates of the fluxes correlated to a strong under-estimate of the $E \times B$ shearing rate. In the particular case of kinetic electrons, a high radial resolution is necessary to finely resolve the structures present near MRSs on the ZF profiles.

In the ITG case, the hybrid model is shown to systematically underestimates the flux levels: $\left\langle\Gamma_{e}^{\text {hyb }}\right\rangle_{t} \approx$ $27 \%\left\langle\Gamma_{e}^{\mathrm{kin}}\right\rangle_{t},\left\langle Q_{e}^{\mathrm{hyb}}\right\rangle_{t} \approx 36 \%\left\langle Q_{e}^{\mathrm{kin}}\right\rangle_{t}$, and $\left\langle Q_{i}^{\mathrm{hyb}}\right\rangle_{t} \approx$ $69 \%\left\langle Q_{i}^{\mathrm{kin}}\right\rangle_{t}$ (flux levels are extrapolated from the radial resolution convergence study). A first cause of this difference is related to the different radial organizations of the ZFs for simulations carried out with the hybrid model and simulations carried out with the fully kinetic model. These differences in the radial organization of the ZFs thus affect the turbulence saturation mechanism and associated transport levels. A second identified shortcoming of the hybrid model is directly related to the hypothesis of an adiabatic response of the passing electrons which consequently leads to ignore their contribution to the fluxes. The hybrid model then fails to reproduce the passing electrons channel of transport which represents, in the ITG case: $63 \%$ and $78 \%$ of particle and electron kinetic energy fluxes, respectively.

In the TEM case, the flux levels of hybrid and kinetic simulations, obtained in the radial resolution convergence study, are different but this difference is not significantly bigger than the uncertainty due to the extrapolation method. Nonetheless, for fully kinetic simulations, a much finer radial resolution was required to converge the fluxes than for the hybrid electron simulations. It shows that the turbulence dynamics and self regulation mechanisms are affected by the passing electron dynamics. Indeed, it could be interesting to carry out simulations of other TEM test cases to see if there are regimes for which the transport level obtained with the hybrid and kinetic electron models diverge more significantly. It should be recalled that not all the TEM cases are dominantly saturated by zonal flows, as mentioned when discussing the choice of temperature ratio for our TEM case. Therefore our results are relevant for particular TEM cases and do not contradict with previous gyrokinetic studies.

\section{ACKNOWLEDGMENT}

The authors would like to thank David Pfefferlé for reading the manuscript, as well as Gabriele Merlo for his help debugging the new hybrid functionality of the GENE code.

Numerical simulations were performed on the Monte Rosa CRAY XE-6 supercomputer of the Swiss National Supercomputing Center, on the HPC-FF cluster of the Jülich Forschungszentrum, and on the IFERC-CSC HE-

\section{LIOS platform.}

The research leading to these results has also received funding from the European Research Council under the European Unions Seventh Framework Programme (FP7/2007-2013)/ERC Grant Agreement No. 277870.

${ }^{1}$ W. Horton, Rev. Mod. Phys. 71, 735 (1999).

${ }^{2}$ T. Tran, K. Appert, M. Fivaz, G. Jost, J. Vaclavik, and L. Villard, Theory of Fusion Plasmas, Int. Workshop (Bologna Editrice Compositori,Società Italiana di Fisica) p. 45 (1999).

${ }^{3}$ S. Jolliet, A. Bottino, P. Angelino, R. Hatzky, T. Tran, B. Mcmillan, O. Sauter, K. Appert, Y. Idomura, and L. Villard, Computer Physics Communications 177, 409 (2007).

${ }^{4}$ Y. Idomura, M. Ida, T. Kano, N. Aiba, and S. Tokuda, Computer Physics Communications 179, 391 (2008).

${ }^{5}$ V. Grandgirard, Y. Sarazin, P. Angelino, A. Bottino, N. Crouseilles, G. Darmet, G. Dif-Pradalier, X. Garbet, P. Ghendrih, S. Jolliet, et al., Plasma Physics and Controlled Fusion 49, B173 (2007).

${ }^{6}$ G. L. Falchetto, J. Vaclavik, and L. Villard, Physics of Plasmas 10, 1424 (2003).

${ }^{7}$ J. Chowdhury, R. Ganesh, P. Angelino, J. Vaclavik, L. Villard, and S. Brunner, Physics of Plasmas 15, 072117 (2008).

${ }^{8}$ R. Waltz, J. Candy, F. Hinton, C. Estrada-Mila, and J. Kinsey, Nuclear Fusion 45, 741 (2005).

${ }^{9} \mathrm{~J}$. Candy, Physics of Plasmas 12, 072307 (2005).

${ }^{10}$ R. E. Waltz, M. E. Austin, K. H. Burrell, and J. Candy, Physics of Plasmas 13, 052301 (2006).

${ }^{11}$ K. Hallatschek and W. Dorland, Phys. Rev. Lett. 95, 055002 (2005).

${ }^{12}$ B. D. Scott, Plasma Physics and Controlled Fusion 48, B277 (2006).

${ }^{13}$ F. Jenko, W. Dorland, M. Kotschenreuther, and B. N. Rogers, Physics of Plasmas 7, 1904 (2000).

${ }^{14}$ T. Görler, X. Lapillonne, S. Brunner, T. Dannert, F. Jenko, F. Merz, and D. Told, Journal of Computational Physics 230, 7053 (2011).

${ }^{15}$ X. Lapillonne, Ph.D. thesis, Ecole Polytechnique Fédérale de Lausanne, Switzerland (2010).

${ }^{16}$ X. Lapillonne, S. Brunner, T. Dannert, S. Jolliet, A. Marinoni, L. Villard, T. Görler, F. Jenko, and F. Merz, Physics of Plasmas 16, 032308 (2009).

${ }^{17}$ J. Dominski, S. Brunner, S. K. Aghdam., T. Görler, F. Jenko, and D. Told, J.Phys:Conf.Ser. 401, 012006 (2012).

18 A. J. Brizard and T. S. Hahm, Rev. Mod. Phys. 79, 421 (2007).

${ }^{19}$ F. Merz, Ph.D. thesis, Universität Münster, Germany (2009).

${ }^{20}$ M. A. Beer, S. C. Cowley, and G. W. Hammett, Physics of Plasmas (1994-present) 2 (1995).

${ }^{21}$ F. Merz and F. Jenko, Phys. Rev. Lett. 100, 035005 (2008).

${ }^{22}$ M. Kammerer, F. Merz, and F. Jenko, Physics of Plasmas (1994present) 15, 052102 (2008).

${ }^{23}$ J. E. Roman, M. Kammerer, F. Merz, and F. Jenko, Parallel Computing 36, 339 (2010), ISSN 0167-8191, parallel Matrix Algorithms and Applications.

${ }^{24}$ F. Merz, C. Kowitz, E. Romero, J. Roman, and F. Jenko, Computer Physics Communications 183, 922 (2012), ISSN 00104655.

${ }^{25}$ F. Romanelli and S. Briguglio, Physics of Fluids B: Plasma Physics 2, 754 (1990).

${ }^{26}$ S. Brunner, Ph.D. thesis, École Polytechnique Fédérale de Lausanne, Switzerland (1997).

${ }^{27}$ Z. Lin, T. S. Hahm, W. W. Lee, W. M. Tang, and R. B. White, Science 281, 1835 (1998).

${ }^{28}$ P. H. Diamond, S.-I. Itoh, K. Itoh, and T. S. Hahm, Plasma Physics and Controlled Fusion 47, R35 (2005).

${ }^{29}$ K. Itoh, S.-I. Itoh, P. H. Diamond, T. S. Hahm, A. Fuj isawa, G. R. Tynan, M. Yagi, and Y. Nagashima, Physics of Plasmas 13, 055502 (2006). 
${ }^{30}$ J. Lang, S. E. Parker, and Y. Chen, Physics of Plasmas 15, 055907 (2008).

${ }^{31}$ T. S. Hahm, Physics of Plasmas 1, 2940 (1994).
${ }^{32}$ T. S. Hahm, M. A. Beer, Z. Lin, G. W. Hammett, W. W. Lee, and W. M. Tang, Physics of Plasmas 6, 922 (1999).

${ }^{33}$ F. L. Hinton and R. D. Hazeltine, Rev. Mod. Phys. 48, 239 (1976). 